\title{
The Heat Semigroup on Configuration Spaces
}

\author{
By \\ Yuri Kondratiev*, Eugene Lytvynov** and Michael RöCKNER ${ }^{* * *}$
}

\begin{abstract}
In this paper, we study properties of the heat semigroup of configuration space analysis. Using a natural "Riemannian-like" structure of the configuration space $\Gamma_{X}$ over a complete, connected, oriented, and stochastically complete Riemannian manifold $X$ of infinite volume, the heat semigroup $\left(e^{-t H^{\Gamma}}\right)_{t \in \mathbb{R}_{+}}$was introduced and studied in [J. Funct. Anal. 154 (1998), 444-500]. Here, $H^{\Gamma}$ is the Dirichlet operator of the Dirichlet form $\mathcal{E}^{\Gamma}$ over the space $L^{2}\left(\Gamma_{X}, \pi_{m}\right)$, where $\pi_{m}$ is the Poisson measure on $\Gamma_{X}$ with intensity $m$-the volume measure on $X$. We construct a metric space $\Gamma_{\infty}$ that is continuously embedded into $\Gamma_{X}$. Under some conditions on the manifold $X$, we prove that $\Gamma_{\infty}$ is a set of full $\pi_{m}$ measure and derive an explicit formula for the heat semigroup: $\left(e^{-t H^{\Gamma}} F\right)(\gamma)=\int_{\Gamma_{\infty}} F(\xi) \mathbf{P}_{t, \gamma}(d \xi)$, where $\mathbf{P}_{t, \gamma}$ is a probability measure on $\Gamma_{\infty}$ for all $t>0, \gamma \in \Gamma_{\infty}$. The central results of the paper are two types of Feller properties for the heat semigroup. The first one is a kind of strong Feller property with respect to the metric on the space $\Gamma_{\infty}$. The second one, obtained in the case $X=\mathbb{R}^{d}$, is the Feller property with respect to the intrinsic metric of the Dirichlet form $\mathcal{E}^{\Gamma}$. Next, we give a direct construction of the independent infinite particle process on the manifold $X$, which is a realization of the Brownian motion on the configuration space. The main point here is that we prove that this process can start in every $\gamma \in \Gamma_{\infty}$, will never leave $\Gamma_{\infty}$, and has continuous sample path in $\Gamma_{\infty}$, provided $\operatorname{dim} X \geq 2$. In this case, we also prove that this process is a strong Markov process whose transition probabilities are given by the $\mathbf{P}_{t, \gamma}(\cdot)$ above. Furthermore, we discuss the necessary changes to be done for constructing the process in the case

Communicated by T. Kawai. Received November 8, 2001.

2000 Mathematics Subject Classification(s): Primary 47D07, 60J60; Secondary 60G57.

*Fakultät für Mathematik, Universität Bielefeld, Postfach 1001 31, D-33501 Bielefeld, Germany; Institute of Mathematics, Kiev, Ukraine; BiBoS, Univ. Bielefeld, Germany. e-mail: kondrat@mathematik.uni-bielefeld.de

**Institut für Angewandte Mathematik, Universität Bonn, Wegelerstr. 6, D-53115 Bonn, Germany; BiBoS, Univ. Bielefeld, Germany. e-mail: lytvynov@wiener.iam.uni-bonn.de

***Fakultät für Mathematik, Universität Bielefeld, Postfach 1001 31, D-33501 Bielefeld, Germany; BiBoS, Univ. Bielefeld, Germany.

e-mail: roeckner@mathematik. uni-bielefeld.de
\end{abstract}


$\operatorname{dim} X=1$. Finally, as an easy consequence we get a "path-wise" construction of the independent particle process on $\Gamma_{\infty}$ from the underlying Brownian motion.

\section{Contents}

$\S 1 . \quad$ Introduction

$\S 2$. Intrinsic Dirichlet Operator on the Poisson Space

$\S 3 . \quad$ Correlation Measures in Configuration Space Analysis

$\S 4$. Heat Kernel Measures $\mathbf{P}_{t, \gamma}$

$\S 5$. Explicit Formula for the Heat Semigroup

$\S 6$. A Strong Feller Property of the Heat Semigroup

$\S 7$. Feller Property of the Heat Semigroup with Respect to the Intrinsic Metric of the Dirichlet Form

$\S 8$. Brownian Motion on the Configuration Space

§9. Appendix: Proof of Lemma 8.1

References

\section{$\S 1 . \quad$ Introduction}

In [3], [4], [5], [6], stochastic analysis and differential geometry on configuration spaces were considerably developed by using the so-called "lifting procedure", see also [33], [28], [34], [35], [1], [2] for further results and reviews.

Let us recall that the configuration space $\Gamma_{X}$ over a complete, connected, oriented, and stochastically complete Riemannian manifold $X$ of infinite volume is defined as the set of all infinite subsets of $X$ which are locally finite. Each configuration $\gamma \in \Gamma_{X}$ can be identified with the Radon measure $\sum_{x \in \gamma} \varepsilon_{x}$. The tangent space to $\Gamma_{X}$ at a point $\gamma \in \Gamma_{X}$, denoted by $T_{\gamma}\left(\Gamma_{X}\right)$, is defined as the direct sum of the tangent spaces to $X$ at $x$, where $x$ runs over the points of the configuration $\gamma$; that is, $T_{\gamma}\left(\Gamma_{X}\right):=\bigoplus_{x \in \gamma} T_{x}(X)$. The gradient $\nabla^{\Gamma} F(\gamma)$ of a differentiable function $F: \Gamma_{X} \rightarrow \mathbb{R}$ at a point $\gamma \in \Gamma_{X}$ is defined as an element of the tangent space $T_{\gamma}\left(\Gamma_{X}\right)$ through a natural lifting of the gradient on $X$. Analogously, one introduces also the notion of divergence of a vector field over $\Gamma_{X}$.

Let $\pi_{m}$ denote the Poisson measure on $\Gamma_{X}$ with intensity $m$ - the volume measure on $X$. By using the integration by parts formula for the Poisson measure, it was shown in [5] that $\pi_{m}$ is a volume measure on $\Gamma_{X}$, in the sense 
that the gradient and the divergence become dual operators on $L^{2}\left(\pi_{m}\right):=$ $L^{2}\left(\Gamma_{X}, \pi_{m}\right)$.

Thus, having identified differentiation and a volume measure on the configuration space, the next step in [5] was to consider the Dirichlet form over $L^{2}\left(\pi_{m}\right)$, which is defined by

$$
\mathcal{E}^{\Gamma}\left(F_{1}, F_{2}\right):=\frac{1}{2} \int_{\Gamma_{X}}\left\langle\nabla^{\Gamma} F_{1}(\gamma), \nabla^{\Gamma} F_{2}(\gamma)\right\rangle_{T_{\gamma}\left(\Gamma_{X}\right)} \pi_{m}(d \gamma)
$$

on an appropriate set of smooth cylinder functions on $\Gamma_{X}$. Using again the integration by parts formula, one obtains the associated Dirichlet operator, i.e., the operator $H^{\Gamma}$ in $L^{2}\left(\pi_{m}\right)$ satisfying $\mathcal{E}^{\Gamma}\left(F_{1}, F_{2}\right)=\left(H^{\Gamma} F_{1}, F_{2}\right)_{L^{2}\left(\pi_{m}\right)}$. This yields, in particular, that the bilinear form $\mathcal{E}^{\Gamma}$ is closable. Moreover, the operator $H^{\Gamma}$ was shown to be essentially selfadjoint. We will preserve the notation $H^{\Gamma}$ for its closure.

The present paper is devoted to the study of properties of the heat semi$\operatorname{group}\left(e^{-t H^{\Gamma}}\right)_{t \in \mathbb{R}_{+}}$.

By using the general theory of Dirichlet forms, it has been already proved in [5], [28] (see also [35]) that there exists a diffusion process (i.e. a strong Markov process with continuous sample paths) on the configuration space that is canonically associated with the heat semigroup $\left(e^{-t H^{\Gamma}}\right)_{t \in \mathbb{R}_{+}}$, i.e., for each $F \in L^{2}\left(\pi_{m}\right)$,

$$
\left(e^{-t H^{\Gamma}} F\right)(\gamma)=\int_{\Omega} F\left(\mathbf{X}_{t}\right) d \mathbf{P}_{\gamma}
$$

for $\pi_{m}$-a.a. (or even quasi-every) $\gamma \in \Gamma_{X}$. This process is then the Brownian motion on $\Gamma_{X}$. Moreover, this process is, in fact, the well-known independent infinite particle process (cf. [5]). The latter is obtained by taking countably many independent Brownian motions on $X$, see [11].

The first part of this paper is devoted to deriving an explicit formula for the heat semigroup. We introduce functionals $B_{n}, n \in \mathbb{N}$, on $\Gamma_{X}$ by

$$
B_{n}(\gamma):=\sum_{x \in \gamma} \exp \left[-\frac{1}{n} \operatorname{dist}\left(x_{0}, x\right)\right],
$$

where $x_{0}$ is a fixed point of the manifold $X$. We define a subset $\Gamma_{\infty}$ of $\Gamma_{X}$ consisting of those configurations $\gamma$ for which $B_{n}(\gamma)<\infty$ for all $n \in \mathbb{N}$, and equip $\Gamma_{\infty}$ with a metric in such a way that the convergence in $\Gamma_{\infty}$ means vague convergence together with convergence of all the functionals $B_{n}$ (see also [21]). Under some conditions on the geometry of the manifold $X$, we prove that $\Gamma_{\infty}$ is of full $\pi_{m}$ measure and that, for each $\gamma \in \Gamma_{\infty}, t>0$, there exists a probability 
measure $\mathbf{P}_{t, \gamma}$ on $\Gamma_{\infty}$ such that for each $F \in L^{2}\left(\Gamma_{\infty}, \pi_{m}\right)$

$$
\left(e^{-t H^{\Gamma}} F\right)(\gamma)=\int_{\Gamma_{\infty}} F(\xi) \mathbf{P}_{t, \gamma}(d \xi), \quad \pi_{m} \text {-a.a. } \gamma \in \Gamma_{\infty} .
$$

To this end, we apply the method for constructing probability measures on $\Gamma_{X}$ described in [39], and define $\mathbf{P}_{t, \gamma}$ via a product measure $\bigotimes_{k=1}^{\infty} p_{t, x_{k}}$ on $X^{\mathbb{N}}$. Here, $p_{t, x}(d y):=p(t, x, y) m(d y), p(t, x, y)$ is the heat kernel of the manifold $X$, and $\gamma=\left\{x_{k}\right\}_{k=1}^{\infty}$ (the resulting measure $\mathbf{P}_{t, \gamma}$ will, however, be independent of the chosen ordering of the points of $\gamma$ ).

The second part of the paper is devoted to our main results which concern two types of Feller properties of the heat semigroup.

We introduce a class $\mathbf{D}$ of measurable functions on $\Gamma_{\infty}$, which particularly contains all bounded local functions, and show that $\mathbf{D}$ is invariant under the action of the semigroup $e^{-t H^{\Gamma}}$. Moreover, we prove that, for each $F \in \mathbf{D}$, the map

$$
\Gamma_{\infty} \ni \gamma \mapsto\left(\mathbf{P}_{t} F\right)(\gamma):=\int_{\Gamma_{\infty}} F(\xi) \mathbf{P}_{t, \gamma}(d \xi) \in \mathbb{R}
$$

is a continuous function on the space $\Gamma_{\infty}\left(\mathbf{P}_{t} F\right.$ is even continuous with respect to some weaker metric). Thus, we obtain a kind of strong Feller property of the heat semigroup. Here, we use results on harmonic analysis over configuration spaces from [18], [19], [22] (see also [23], [24], [25], [26], [7]),

Next, we consider a metric space $\ddot{\Gamma}_{\infty}$ which is an appropriate extension of $\Gamma_{\infty}$ to multiple configurations in $X$, i.e., to $\mathbb{Z}_{+}$-valued Radon measures on $X$. Restricting ourselves to the case $X=\mathbb{R}^{d}$, we prove that the operators $\left(\mathbf{P}_{t}\right)_{t>0}$ defined by

$$
\ddot{\Gamma}_{\infty} \ni \gamma \mapsto\left(\mathbf{P}_{t} F\right)(\gamma):=\int_{\ddot{\Gamma_{\infty}}} F(\xi) \mathbf{P}_{t, \gamma}(d \xi) \in \mathbb{R}
$$

preserve the class of all bounded functions on $\ddot{\Gamma}_{\infty}$ which are continuous with respect to the intrinsic metric of the Dirichlet form $\mathcal{E}^{\Gamma}$ (see [34]). Thus, for this metric, we have the usual Feller property of the heat semigroup.

In the third part of the paper, which is more probabilistic, we present a direct construction of the independent infinite particle process on the manifold $X$ with the state space $\ddot{\Gamma}_{\infty}$, which will be therefore a realization of the Brownian motion on the configuration space mentioned above. We show that, if the dimension of $X$ is $\geq 2$, the constructed process is the unique continuous strong Markov process on $\Gamma_{\infty}$ whose transition probabilities are given by $\mathbf{P}_{t}(\gamma, \cdot):=$ $\mathbf{P}_{t, \gamma}(\cdot)$. In particular, it starts at any configuration in $\Gamma_{\infty}$ and never leaves $\Gamma_{\infty}$. If $\operatorname{dim} X=1$, one cannot exclude collisions of the particles, but it is still possible 
to realize the Brownian motion on the configuration space as a continuous Markov process on $\ddot{\Gamma}_{\infty}$. Finally, we describe a path-wise construction of the infinite particle process starting from any point in $\Gamma_{\infty}$ (respectively $\ddot{\Gamma}_{\infty}$ ). More precisely, we show that the obvious heuristic construction can be performed rigorously.

We should mention that independent infinite particle processes have been studied by many authors, see e.g. [36], but neither in this paper, nor in any other reference we are aware of, it was proved that the process takes values in the configuration space for all values of $t>0$.

\section{$\S 2$. Intrinsic Dirichlet Operator on the Poisson Space}

In this section, we will briefly recall the definition and some properties of the intrinsic Dirichlet operator on the Poisson space. We refer the reader to [5], [3], [4] for details and proofs.

Let $X$ be a complete, connected, oriented $C^{\infty}$ Riemannian manifold. Let $m$ denote the volume measure on $X$, and we suppose that $m(X)=\infty$. Let $\nabla^{X}$ and $H^{X}:=-(1 / 2) \Delta^{X}$ be the gradient and Laplace-Beltrami operator on $X$, respectively. We denote by $\mathcal{D}:=C_{0}^{\infty}(X)$ the space of all $C^{\infty}$ functions on $X$ with compact support. It is well-known that $\left(H^{X}, \mathcal{D}\right)$ is essentially selfadjoint on $L^{2}(m):=L^{2}(X, \mathcal{B}(X), m)$, where $\mathcal{B}(X)$ is the Borel $\sigma$-algebra on $X$. In what follows, we will always suppose that $H^{X}$ is conservative (cf. e.g. [38]).

Let $p(t, x, y), t \in(0, \infty), x, y \in X$, denote the heat kernel of the operator $H^{X}$ :

$$
\left(e^{-t H^{X}} \varphi\right)(x)=\int_{X} \varphi(y) p(t, x, y) m(d y), \quad m \text {-a.e. } x \in X,
$$

where $\varphi$ is a bounded measurable function on $X$. We recall that $p(t, x, y)$ is a strictly positive $C^{\infty}$ function on $(0, \infty) \times X \times X$ (cf. e.g. [10]).

The conservativity condition yields, in particular, that

$$
\int_{X} p(t, x, y) m(d y)=1, \quad t \in(0, \infty), x \in X,
$$

i.e., for each $t>0$ and $x \in X$ the heat kernel determines a probability measure

$$
p_{t, x}(d y):=p_{t}(x, d y)=p(t, x, y) m(d y)
$$

on $X$. Thus, the manifold $X$ is stochastically complete.

Next, we consider the configuration space $\Gamma_{X}$ over $X$ - the set of all infinite subsets in $X$ which are locally finite:

$$
\Gamma_{X}:=\left\{\gamma \subset X|| \gamma \mid=\infty \text { and }\left|\gamma_{\Lambda}\right|<\infty \text { for each compact } \Lambda \subset X\right\} .
$$


Here, $|\cdot|$ denotes the cardinality of a set and $\gamma_{\Lambda}:=\gamma \cap \Lambda$. One can identify any $\gamma \in \Gamma_{X}$ with the positive Radon measure

$$
\sum_{x \in \gamma} \varepsilon_{x} \in \mathcal{M}(X)
$$

where $\mathcal{M}(X)$ stands for the set of all positive Radon measures on $\mathcal{B}(X)$. The space $\Gamma_{X}$ can be endowed with the relative topology as a subset of the space $\mathcal{M}(X)$ with the vague topology, i.e., the weakest topology on $\Gamma_{X}$ with respect to which all maps

$$
\Gamma_{X} \ni \gamma \mapsto\langle\varphi, \gamma\rangle:=\int_{X} \varphi(x) \gamma(d x)=\sum_{x \in \gamma} \varphi(x), \quad \varphi \in \mathcal{D},
$$

are continuous. We shall denote the Borel $\sigma$-algebra on $\Gamma_{X}$ by $\mathcal{B}\left(\Gamma_{X}\right)$.

Let $\pi_{m}$ denote the Poisson measure on $\left(\Gamma_{X}, \mathcal{B}\left(\Gamma_{X}\right)\right)$ with intensity $m$. This measure can be characterized by its Laplace transform

$$
\ell_{\pi_{m}}(\varphi):=\int_{\Gamma_{X}} e^{\langle\varphi, \gamma\rangle} \pi_{m}(d \gamma)=\exp \left(\int_{X}\left(e^{\varphi(x)}-1\right) m(d x)\right), \quad \varphi \in \mathcal{D} .
$$

We refer to e.g. [39], [37], [5] for a detailed discussion of the construction of the Poisson measure on the configuration space. Now, we recall how to define the intrinsic Dirichlet operator $H^{\Gamma}$ in the space $L^{2}\left(\pi_{m}\right):=L^{2}\left(\Gamma_{X}, \mathcal{B}\left(\Gamma_{X}\right), \pi_{m}\right)$.

Let $T_{x}(X)$ denote the tangent space to $X$ at a point $x \in X$. The tangent space to $\Gamma_{X}$ at a point $\gamma \in \Gamma_{X}$ is defined as the Hilbert space

$$
T_{\gamma}\left(\Gamma_{X}\right):=\bigoplus_{x \in \gamma} T_{x}(X)
$$

Thus, each $V(\gamma) \in T_{\gamma}\left(\Gamma_{X}\right)$ has the form $V(\gamma)=(V(\gamma, x))_{x \in \gamma}$, where $V(\gamma, x) \in$ $T_{x}(X)$, and

$$
\|V(\gamma)\|_{T_{\gamma}\left(\Gamma_{X}\right)}^{2}=\sum_{x \in \gamma}\|V(\gamma, x)\|_{T_{x}(X)}^{2}
$$

Let $\gamma \in \Gamma_{X}$ and $x \in \gamma$. We denote by $\mathcal{O}_{\gamma, x}$ an arbitrary open neighborhood of $x$ in $X$ such that $\mathcal{O}_{\gamma, x} \cap(\gamma \backslash\{x\})=\varnothing$. Now, for a function $F: \Gamma_{X} \rightarrow \mathbb{R}$, $\gamma \in \Gamma_{X}$, and $x \in \gamma$, we define a function $F_{x}(\gamma, \cdot): \mathcal{O}_{\gamma, x} \rightarrow \mathbb{R}$ by

$$
\mathcal{O}_{\gamma, x} \ni y \mapsto F_{x}(\gamma, y):=F((\gamma \backslash\{x\}) \cup\{y\}) \in \mathbb{R} .
$$

We say that a function $F: \Gamma_{X} \rightarrow \mathbb{R}$ is differentiable at $\gamma \in \Gamma_{X}$ if for each $x \in \gamma$ the function $F_{x}(\gamma, \cdot)$ is differentiable at $x$ and

$$
\nabla^{\Gamma} F(\gamma):=\left(\nabla^{X} F_{x}(\gamma, x)\right)_{x \in \gamma} \in T_{\gamma}\left(\Gamma_{X}\right)
$$


where

$$
\nabla^{X} F_{x}(\gamma, x):=\left.\nabla_{y}^{X} F_{x}(\gamma, y)\right|_{y=x}
$$

(cf. [1], [2]). Evidently, this definition is independent of the choice of the set $\mathcal{O}_{\gamma, x}$. We will call $\nabla^{\Gamma} F(\gamma)$ the gradient of $F$ at $\gamma$.

We introduce the set $\mathcal{F} C_{\mathrm{b}}^{\infty}\left(\mathcal{D}, \Gamma_{X}\right)$ consisting of all smooth cylinder functions on $\Gamma_{X}$, i.e., all functions of the form

$$
F(\gamma)=g_{F}\left(\left\langle\varphi_{1}, \gamma\right\rangle, \ldots,\left\langle\varphi_{N}, \gamma\right\rangle\right), \quad \gamma \in \Gamma_{X}
$$

where $N \in \mathbb{N}, \varphi_{1}, \ldots, \varphi_{N} \in \mathcal{D}$, and $g_{F} \in C_{\mathrm{b}}^{\infty}\left(\mathbb{R}^{N}\right)$. Any function $F$ of the form (2.6) is differentiable at each point $\gamma \in \Gamma_{X}$, and its gradient is given by

$$
\left(\nabla^{\Gamma} F\right)(\gamma)=\sum_{j=1}^{N} \partial_{j} g_{F}\left(\left\langle\varphi_{1}, \gamma\right\rangle, \ldots,\left\langle\varphi_{N}, \gamma\right\rangle\right) \nabla^{X} \varphi_{j}
$$

where $\partial_{j} g_{F}$ means derivative with respect to the $j$-th coordinate.

Then, the corresponding pre-Dirichlet form is

$$
\mathcal{E}^{\Gamma}(F, G):=\frac{1}{2} \int_{\Gamma_{X}}\left\langle\nabla^{\Gamma} F(\gamma), \nabla^{\Gamma} G(\gamma)\right\rangle_{T_{\gamma}\left(\Gamma_{X}\right)} \pi_{m}(d \gamma), \quad F, G \in \mathcal{F} C_{\mathrm{b}}^{\infty}\left(\mathcal{D}, \Gamma_{X}\right) .
$$

By using the integration by parts formula on the Poisson space, one shows that the associated Dirichlet operator $H^{\Gamma}$, i.e., the operator satisfying

$$
\mathcal{E}^{\Gamma}(F, G)=\left(H^{\Gamma} F, G\right)_{L^{2}\left(\pi_{m}\right)}, \quad F, G \in \mathcal{F} C_{\mathrm{b}}^{\infty}\left(\mathcal{D}, \Gamma_{X}\right),
$$

is of the form

$$
\begin{aligned}
\left(H^{\Gamma} F\right)(\gamma)= & -\sum_{i, j=1}^{N} \partial_{i} \partial_{j} g_{F}\left(\left\langle\varphi_{1}, \gamma\right\rangle, \ldots,\left\langle\varphi_{N}, \gamma\right\rangle\right) \\
& \times \int_{X} \frac{1}{2}\left\langle\nabla^{X} \varphi_{i}(x), \nabla^{X} \varphi_{j}(x)\right\rangle_{T_{x}(X)} \gamma(d x) \\
& +\sum_{j=1}^{N} \partial_{j} g_{F}\left(\left\langle\varphi_{1}, \gamma\right\rangle, \ldots,\left\langle\varphi_{N}, \gamma\right\rangle\right) \int_{X}\left(H^{X} \varphi_{j}\right)(x) \gamma(d x),
\end{aligned}
$$

where $F$ is given by (2.6). Therefore, the bilinear form $\left(\mathcal{E}^{\Gamma}, \mathcal{F} C_{\mathrm{b}}^{\infty}\left(\mathcal{D}, \Gamma_{X}\right)\right)$ is closable on $L^{2}\left(\pi_{m}\right)$, and with its closure we can associate a positive definite selfadjoint operator, the Friedrichs extension of $H^{\Gamma}$, which will be also denoted 
by $H^{\Gamma}$. (In fact, $\mathcal{F} C_{\mathrm{b}}^{\infty}\left(\mathcal{D}, \Gamma_{X}\right)$ is a domain of essential selfadjointness of $H^{\Gamma}$, see [5, Theorem 5.3].)

Consider the corresponding heat semigroup $\left(e^{-t H^{\Gamma}}\right)_{t \in \mathbb{R}_{+}}$in $L^{2}\left(\pi_{m}\right)$, where as usual $\mathbb{R}_{+}:=[0, \infty)$. We set

$$
E\left(\mathcal{D}_{1}, \Gamma\right):=\text { l.h. }\left\{\exp [\langle\log (1+\varphi), \cdot\rangle] \mid \varphi \in \mathcal{D}_{1}\right\} .
$$

Here, l.h. means linear hull and

$$
\begin{array}{r}
\mathcal{D}_{1}:=\left\{\varphi \in D\left(H^{X}\right) \cap L^{1}(m) \mid H^{X} \varphi \in L^{1}(m)\right. \text { and } \\
-\delta \leq \varphi \leq 0 \text { for some } \delta \in(0,1)\} .
\end{array}
$$

Proposition 2.1. We have

$$
\begin{gathered}
e^{-t H^{\Gamma}} \exp [\langle\log (1+\varphi), \cdot\rangle]=\exp \left[\left\langle\log \left(1+e^{-t H^{X}} \varphi\right), \cdot\right\rangle\right] \\
\pi_{m} \text {-a.e. for all } \varphi \in \mathcal{D}_{1}
\end{gathered}
$$

Proof. See [5, Proposition 4.1].

As a direct consequence of this proposition, in particular, one obtains that $\left(H^{\Gamma}, E\left(\mathcal{D}_{1}, \Gamma\right)\right)$ is essentially selfadjoint on $L^{2}\left(\pi_{m}\right)$.

Finally, the diffusion process that is properly associated with the Dirichlet form $\left(\mathcal{E}^{\Gamma}, \operatorname{Dom}\left(\mathcal{E}^{\Gamma}\right)\right)$ is the usual independent infinite particle process, or in other terms, Brownian motion on $\Gamma_{X}$ (cf. [5, Subsection 6.2]).

\section{§3. Correlation Measures in Configuration Space Analysis}

In this section, we shall recall some facts on $K$-transforms and correlation measures. We shall follow [18], [22] (see also [23], [24], [25], [26], [19], [20], [7]; in [18], [22], [7] the reader can also find many further references and historical comments).

Denote by $\Gamma_{X, 0}$ the space of all finite configurations over $X$ :

$$
\Gamma_{X, 0}:=\bigsqcup_{n=0}^{\infty} \Gamma_{X}^{(n)}, \quad \Gamma_{X}^{(0)}=\{\varnothing\}, \quad \Gamma_{X}^{(n)}=\{\eta \subset X|| \eta \mid=n\}, \quad n \in \mathbb{N} .
$$

Let

$$
\tilde{X}^{n}=\left\{\left(x_{1}, \ldots, x_{n}\right) \in X^{n} \mid x_{i} \neq x_{j} \text { when } i \neq j\right\}
$$

and let $S_{n}$ denote the group of permutations of $\{1, \ldots, n\}$, which acts on $\tilde{X}^{n}$ by

$$
\sigma\left(x_{1}, \ldots, x_{n}\right)=\left(x_{\sigma(1)}, \ldots, x_{\sigma(n)}\right), \quad \sigma \in S_{n} .
$$


Through the natural bijection

$$
\widetilde{X}^{n} / S_{n} \mapsto \Gamma_{X}^{(n)}
$$

one defines a topology on $\Gamma_{X}^{(n)}$. The space $\Gamma_{X, 0}$ is then equipped with the topology of disjoint unions. Let $\mathcal{B}\left(\Gamma_{X, 0}\right)$ denote the corresponding Borel $\sigma$ algebra. A set $K \subset \Gamma_{X, 0}$ is compact if there exists $N \in \mathbb{N}$ with $K \cap \Gamma_{X}^{(n)}=\varnothing$ for all $n>N$ and $K \cap \Gamma_{X}^{(n)}$ is compact for all $n \leq N$. The set of all Borel sets in $\Gamma_{X, 0}$ with compact closure is denoted by $\mathcal{B}_{\mathrm{c}}\left(\Gamma_{X, 0}\right)$.

A $\mathcal{B}\left(\Gamma_{X, 0}\right)$-measurable function $G: \Gamma_{X, 0} \rightarrow \mathbb{R}$ is said to have bounded support if there exist a relatively compact open set $\Lambda \subset X$ and $N \in \mathbb{N}$ such that $\{G \neq 0\} \subset \bigsqcup_{n=0}^{N} \Gamma_{\Lambda}^{(n)}$. The space of bounded functions on $\Gamma_{X, 0}$ with bounded support is denoted by $B_{\mathrm{bs}}\left(\Gamma_{X, 0}\right)$.

In what follows, for any $\gamma \in \Gamma_{X}$, we shall use the notation $\sum_{\eta \Subset \gamma}$ for the summation over all $\eta \subset \gamma$ such that $|\eta|<\infty$. For a function $G: \Gamma_{X, 0} \rightarrow \mathbb{R}$, the $K$-transform of $G$ is then defined by

$$
(K G)(\gamma):=\sum_{\eta \Subset \gamma} G(\eta)
$$

for each $\gamma \in \Gamma_{X}$ such that at least one of the series $\sum_{\eta \Subset \gamma} G^{+}(\eta)$ or $\sum_{\eta \Subset \gamma} G^{-}(\eta)$ converges in $\mathbb{R}_{+}$, where $G^{+}(\gamma):=\max \{0, G(\gamma)\}, G^{-}(\gamma)=-\min \{0, G(\gamma)\}$. For each $G \in B_{\mathrm{bs}}\left(\Gamma_{X, 0}\right)$ and each $\gamma \in \Gamma_{X}$, the series $\sum_{\eta \Subset \gamma} G(\eta)$ is always finite, and moreover, $(K G)(\cdot)$ is a $\mathcal{B}\left(\Gamma_{X}\right)$-measurable function on $\Gamma_{X}$ (cf. [18, Proposition 3.5]).

Let $\mu$ be a probability measure on $\left(\Gamma_{X}, \mathcal{B}\left(\Gamma_{X}\right)\right)$. The correlation measure corresponding to $\mu$ is defined by

$$
\rho_{\mu}(A):=\int_{\Gamma_{X}}\left(K \mathbf{1}_{A}\right)(\gamma) \mu(d \gamma), \quad A \in \mathcal{B}\left(\Gamma_{X, 0}\right)
$$

$\rho_{\mu}$ is obviously a measure on $\left(\Gamma_{X, 0}, \mathcal{B}\left(\Gamma_{X, 0}\right)\right)$.

Proposition 3.1. Let $\mu$ be a probability measure on $\left(\Gamma_{X}, \mathcal{B}\left(\Gamma_{X}\right)\right)$. Then, the measure $\rho_{\mu}$ is locally finite, i.e.,

$$
\rho_{\mu}(A)<\infty \text { for all } A \in \mathcal{B}_{\mathrm{c}}\left(\Gamma_{X, 0}\right),
$$

if and only if

$$
\int_{\Gamma_{X}}\left|\gamma_{\Lambda}\right|^{n} \mu(d \gamma)<\infty \quad \text { for all } n \in \mathbb{N} \text { and } \Lambda \in \mathcal{B}_{c}(X) .
$$


Proof. See [18, Proposition 4.2].

We say that a measure $\mu$ satisfying (3.4) has finite local moments and denote the set of all such measures on $\left(\Gamma_{X}, \mathcal{B}\left(\Gamma_{X}\right)\right)$ by $\mathcal{M}_{\mathrm{fm}}\left(\Gamma_{X}\right)$. The set of all locally finite measures on $\Gamma_{X, 0}$ will be denoted by $\mathcal{M}_{\mathrm{lf}}\left(\Gamma_{X, 0}\right)$.

Proposition 3.2. Let $\mu \in \mathcal{M}_{\mathrm{fm}}\left(\Gamma_{X}\right)$ and let $G: \Gamma_{X, 0} \rightarrow \mathbb{R}$ be a measurable function which is integrable with respect to the measure $\rho_{\mu}$. Then, $K G$ is well-defined and finite $\mu$-a.e., and integrable with respect to the measure $\mu$. If for some $G^{\prime}: \Gamma_{X, 0} \rightarrow \mathbb{R}, G=G^{\prime} \rho_{\mu}$-a.e., then $K G=K G^{\prime} \mu$-a.e., and hence the $K$-transform defines a linear mapping

$$
K: L^{1}\left(\Gamma_{X, 0}, \mathcal{B}\left(\Gamma_{X, 0}\right), \rho_{\mu}\right) \rightarrow L^{1}\left(\Gamma_{X}, \mathcal{B}\left(\Gamma_{X}\right), \mu\right)
$$

Furthermore, we have

$$
\|K G\|_{L^{1}(\mu)} \leq\|K|G|\|_{L^{1}(\mu)}=\|G\|_{L^{1}\left(\rho_{\mu}\right)}
$$

and

$$
\int_{\Gamma_{X}, 0} G(\eta) \rho_{\mu}(d \eta)=\int_{\Gamma_{X}}(K G)(\gamma) \mu(d \gamma)
$$

Proof. See [18, Theorem 4.11].

For two functions $G_{1}, G_{2}: \Gamma_{X, 0} \rightarrow \mathbb{R}$, the $\star$-convolution of $G_{1}$ and $G_{2}$ is defined as the mapping $G_{1} \star G_{2}: \Gamma_{X, 0} \rightarrow \mathbb{R}$ given by

$$
\left(G_{1} \star G_{2}\right)(\eta):=\sum_{\left(\eta_{1}, \eta_{2}, \eta_{3}\right) \in \mathcal{P}_{3}(\eta)} G_{1}\left(\eta_{1} \cup \eta_{2}\right) G_{2}\left(\eta_{2} \cup \eta_{3}\right),
$$

where $\mathcal{P}_{3}(\eta)$ denotes the set of all ordered partitions $\left(\eta_{1}, \eta_{2}, \eta_{3}\right)$ of $\eta$ into 3 parts. Clearly, if $G_{1}, G_{2}$ are $\mathcal{B}\left(\Gamma_{X, 0}\right)$-measurable, then so is $G_{1} \star G_{2}$. The main property of the $\star$-convolution is given by the following formula (see $[18$, Proposition 3.11]):

$$
\left(K\left(G_{1} \star G_{2}\right)\right)(\gamma)=\left(K G_{1}\right)(\gamma) \cdot\left(K G_{2}\right)(\gamma),
$$

provided $\left(K G_{1}\right)(\gamma)$ and $\left(K G_{2}\right)(\gamma)$ exist.

Let $\sigma$ be a non-atomic Radon measure. The Lebesgue-Poisson measure $\lambda_{\sigma}$ on $\left(\Gamma_{X, 0}, \mathcal{B}\left(\Gamma_{X, 0}\right)\right)$ with intensity $\sigma$ is defined by

$$
\lambda_{\sigma}:=\varepsilon_{\varnothing}+\sum_{n=1}^{\infty} \frac{1}{n !} \sigma^{\otimes n}
$$


where the measure $\sigma^{\otimes n}$ is defined on $\Gamma_{X}^{(n)}$ via the bijection (3.1).

Finally, let us introduce the notion of correlation functions. Suppose that a measure $\rho \in \mathcal{M}_{\mathrm{lf}}\left(\Gamma_{X, 0}\right)$ is absolutely continuous with respect to the LebesguePoisson measure $\lambda_{m}$ with intensity $m$, and define the functions $k^{(n)}: \Gamma_{X}^{(n)} \rightarrow \mathbb{R}$ as the restrictions of the Radon-Nikodym derivative $k:=d \rho / d \lambda_{m}$ to $\Gamma_{X}^{(n)}$. In the case where $\rho=\rho_{\mu}$ is a correlation measure, the functions $\left(k_{\mu}^{(n)}\right)_{n=1}^{\infty}$ are called correlation functions of the measure $\mu$.

\section{§4. Heat Kernel Measures $\mathbf{P}_{t, \gamma}$}

In this section, we shall construct a family of probability measures $\mathbf{P}_{t, \gamma}$ on the configuration space so that $\mathbf{P}_{t, \bullet}(\cdot)$ is the kernel of the integral operator $e^{-t H^{\Gamma}}$.

First, we recall the construction of probability measures on the configuration space $\Gamma_{X}$ proposed by A. M. Vershik et al. [39], see also [16].

Let us consider the infinite product $X^{\mathbb{N}}=\underset{k=1}{\times} X_{k}, X_{k}=X$, furnished with the product topology, and let $\mathcal{B}\left(X^{\mathbb{N}}\right)$ denote the Borel $\sigma$-algebra on $X^{\mathbb{N}}$. We define $\widetilde{X}^{\mathbb{N}}$ as the set of all elements $\left(x_{1}, x_{2}, \ldots\right) \in X^{\mathbb{N}}$ such that 1) $x_{i} \neq x_{j}$ when $i \neq j$, and 2) the sequence $\left\{x_{k}\right\}_{k=1}^{\infty}$ has no accumulation points in $X$. Evidently,

$$
\begin{aligned}
\tilde{X}^{\mathbb{N}}= & {\left[\bigcap_{i \neq j}\left\{\left(x_{1}, x_{2}, \ldots\right) \in X^{\mathbb{N}}: x_{i} \neq x_{j}\right\}\right] } \\
& \cap\left[\bigcap_{n=1}^{\infty} \bigcup_{k=1}^{\infty}\left\{\left(x_{1}, x_{2}, \ldots\right) \in X^{\mathbb{N}}: \forall l \geq k d\left(x_{0}, x_{l}\right) \geq n\right\}\right]
\end{aligned}
$$

where $x_{0}$ is a fixed point of $X$ and $d(\cdot, \cdot)$ denotes the distance on $X$. Hence, $\widetilde{X}^{\mathbb{N}} \in \mathcal{B}\left(X^{\mathbb{N}}\right)$.

Let $\nu_{k}, k \in \mathbb{N}$, be nonatomic probability measures on $(X, \mathcal{B}(X))$ and consider the product measure $\nu:=\bigotimes_{k=1}^{\infty} \nu_{k}$ on $\left(X^{\mathbb{N}}, \mathcal{B}\left(X^{\mathbb{N}}\right)\right)$. (4.1) and the BorelCantelli lemma imply the following:

Lemma 4.1. [39] We have $\nu\left(\widetilde{X}^{\mathbb{N}}\right)=0$ or 1 , and $\nu\left(\widetilde{X}^{\mathbb{N}}\right)=1$ if and only if

$$
\sum_{k=1}^{\infty} \nu_{k}(\Lambda)<\infty \quad \text { for each compact } \Lambda \subset X \text {. }
$$


Let $S_{\infty}$ denote the group of all permutations of the sequence of natural numbers, which acts on $X^{\mathbb{N}}$ :

$$
\sigma\left(y_{1}, y_{2}, \ldots\right)=\left(y_{\sigma(1)}, y_{\sigma(2)}, \ldots\right), \quad \sigma \in S_{\infty} .
$$

The space $\widetilde{X}^{\mathbb{N}}$ is invariant under the action of $S_{\infty}$. Through the natural bijection $\widetilde{X}^{\mathbb{N}} / S_{\infty} \mapsto \Gamma_{X}$, we shall identify these two spaces. Let $I: \widetilde{X}^{\mathbb{N}} \rightarrow \Gamma_{X}$ be given by

$$
\widetilde{X}^{\mathbb{N}} \ni \mathbf{x}=\left(x_{1}, x_{2}, \ldots\right) \mapsto I \mathbf{x}=\left\{x_{1}, x_{2}, \ldots\right\} \in \Gamma_{X}
$$

Thus, $I$ maps an element $\mathbf{x} \in \widetilde{X}^{\mathbb{N}}$ into the corresponding equivalence class $[\mathbf{x}] \in \widetilde{X}^{\mathbb{N}} / S_{\infty}$.

The mapping $I: \widetilde{X}^{\mathbb{N}} \rightarrow \Gamma_{X}$ defined by (4.3) is $\mathcal{B}\left(\widetilde{X}^{\mathbb{N}}\right)-\mathcal{B}\left(\Gamma_{X}\right)$-measurable (here $\mathcal{B}\left(\widetilde{X}^{\mathbb{N}}\right)$ denotes the trace $\sigma$-algebra of $\mathcal{B}\left(X^{\mathbb{N}}\right)$ on $\left.\widetilde{X}^{\mathbb{N}}\right)$. Indeed, the $\sigma$ algebra $\mathcal{B}\left(\Gamma_{X}\right)$ is generated by the sets of the form

$$
A_{\Lambda, n}=\left\{\gamma \in \Gamma_{X}:\left\langle\mathbf{1}_{\Lambda}, \gamma\right\rangle=n\right\},
$$

where $n \in \mathbb{Z}_{+}:=\mathbb{N} \cup\{0\}, \Lambda$ is a compactum in $X$, and $\mathbf{1}_{\Lambda}$ is the indicator of $\Lambda$ (see e.g. [17], [29]). Then,

$$
\begin{aligned}
I^{-1}\left(A_{\Lambda, n}\right) & =\bigcup_{\sigma \in S_{\infty}^{\mathrm{fin}}}\left\{\left(x_{1}, x_{2}, \ldots\right)\right. \\
& \left.\in \widetilde{X}^{\mathbb{N}}: x_{\sigma(i)} \in \Lambda, i=1, \ldots, n, x_{\sigma(i)} \in \Lambda^{\mathrm{c}}, i \geq n+1\right\},
\end{aligned}
$$

where $S_{\infty}^{\text {fin }}$ denotes the group of all finite permutations of the sequence of natural numbers, and $\Lambda^{\mathrm{c}}:=X \backslash \Lambda$. Since $S_{\infty}^{\text {fin }}$ has a countable number of elements, we conclude from (4.4) that $I^{-1}\left(A_{\Lambda, n}\right) \in \mathcal{B}\left(\widetilde{X}^{\mathbb{N}}\right)$, which implies the measurability of $I$.

Hence, if the measures $\nu_{k}, k \in \mathbb{N}$, satisfy condition (4.2), we can consider the image of the probability measure $\nu$ on $\widetilde{X}^{\mathbb{N}}$ under the mapping $I$, which is a probability measure on $\Gamma_{X}$. Evidently, this image-measure is independent of the order of the $\nu_{k}$ 's, that is, it coincides with the measure on $\Gamma_{X}$ constructed through the product-measure $\bigotimes_{k=1}^{\infty} \nu_{\sigma(k)}$ for each $\sigma \in S_{\infty}$.

Let now $t>0$ and let $\gamma$ be a fixed point of $\Gamma_{X}$ such that

$$
\sum_{x \in \gamma} p_{t, x}(\Lambda)<\infty \text { for each compact } \Lambda \subset X,
$$

where $p_{t, x}$ is as in (2.3). Define

$$
\mathbf{P}_{t, \gamma}:=\mathbb{P}_{t, \mathbf{x}} \circ I^{-1}, \text { where } \mathbb{P}_{t, \mathbf{x}}:=\bigotimes_{k=1}^{\infty} p_{t, x_{k}}
$$


and where $\mathbf{x}=\left(x_{k}\right)_{k=1}^{\infty} \in \widetilde{X}^{\mathbb{N}}$ is an arbitrary element of the set $I^{-1}\{\gamma\}$ (the resulting measure $\mathbf{P}_{t, \gamma}$ being independent of the choice of $\mathbf{x}$ ).

Below, we shall need the correlation measure $\rho_{t, \gamma}$ of $\mathbf{P}_{t, \gamma}$.

Proposition 4.1. Let $t>0$ and let $\gamma \in \Gamma_{X}$ satisfy (4.5). Then, the correlation measure $\rho_{t, \gamma}$ of $\mathbf{P}_{t, \gamma}$ is given by

$$
\begin{aligned}
& \rho_{t, \gamma} \uparrow \Gamma_{X}^{(0)}:=\rho_{t, \gamma}^{(0)}:=\varepsilon_{\varnothing}, \\
& \rho_{t, \gamma}\left\lceil\Gamma_{X}^{(n)}:=\rho_{t, \gamma}^{(n)}:=\sum_{\theta \Subset \gamma:|\theta|=n}\left(\otimes_{x \in \theta} p_{t, x}\right) \circ T_{n}^{-1}, \quad n \in \mathbb{N},\right.
\end{aligned}
$$

where $T_{n}: \widetilde{X}^{n} \rightarrow \Gamma_{X}^{(n)}$ is the composition of the natural quotient map $\widetilde{X}^{n} \rightarrow$ $\widetilde{X}^{n} / S_{n}$ and the bijection (3.1) (the measure $\left(\otimes_{x \in \theta} p_{t, x}\right) \circ T_{n}^{-1}$ is independent of a chosen order of the product of the measures $\left.p_{t, x}\right)$. Moreover, we have

$$
\rho_{t, \gamma}\left(\Gamma_{\Lambda}^{(n)}\right) \leq \frac{1}{n !}\left(\sum_{x \in \gamma} p_{t, x}(\Lambda)\right)^{n}<\infty \quad \text { for each compact } \Lambda \subset X
$$

in particular, $\rho_{t, \gamma} \in \mathcal{M}_{\mathrm{lf}}\left(\Gamma_{X, 0}\right)$ and $\mathbf{P}_{t, \gamma} \in \mathcal{M}_{\mathrm{fm}}\left(\Gamma_{X}\right)$.

Proof. Let the measure $\rho_{t, \gamma}$ on $\left(\Gamma_{X, 0}, \mathcal{B}\left(\Gamma_{X, 0}\right)\right)$ be defined by (4.7). For a measurable function $G: \Gamma_{X, 0} \rightarrow \mathbb{R}$, we have $G=\left(G^{(n)}\right)_{n=0}^{\infty}$, where $G^{(n)}:=$ $G \uparrow \Gamma_{X}^{(n)}$. Then, by using the definition of $\mathbf{P}_{t, \gamma}$ and the monotone convergence theorem, we have, for any $\mathcal{B}\left(\Gamma_{X, 0}\right)$-measurable function $G: \Gamma_{X, 0} \rightarrow \mathbb{R}_{+}$, that

$$
\begin{aligned}
\int_{\Gamma_{X}}(K G)(\xi) \mathbf{P}_{t, \gamma}(d \xi)= & \int_{\widetilde{X}^{\mathbb{N}}}(K G)(I \mathbf{y}) \mathbb{P}_{t, \mathbf{x}}(d \mathbf{y}) \\
= & G^{(0)}(\varnothing)+\sum_{n=1}^{\infty} \sum_{\left\{i_{1}, \ldots, i_{n}\right\} \subset\{1,2, \ldots\}} \int_{\widetilde{X}^{\mathbb{N}}} G^{(n)} \\
& \circ T_{n}\left(y_{i_{1}}, \ldots, y_{i_{n}}\right) \bigotimes_{k=1}^{\infty} p_{t, x_{k}}\left(d y_{k}\right) \\
= & G^{(0)}(\varnothing)+\sum_{n=1}^{\infty} \sum_{\left\{i_{1}, \ldots, i_{n}\right\} \subset\{1,2, \ldots\}} \int_{\widetilde{X}^{n}} G^{(n)} \\
& \circ T_{n}\left(y_{1}, \ldots, y_{n}\right) p_{t, x_{i_{1}}} \otimes \cdots \otimes p_{t, x_{i_{n}}}\left(d y_{1}, \ldots, d y_{n}\right) \\
= & G^{(0)}(\varnothing)+\sum_{n=1}^{\infty} \int_{\widetilde{X}^{n}} G^{(n)} \circ T_{n}\left(y_{1}, \ldots, y_{n}\right)
\end{aligned}
$$




$$
\begin{aligned}
& \times \sum_{\left\{i_{1}, \ldots, i_{n}\right\} \subset\{1,2, \ldots\}} p_{t, x_{i_{1}}} \otimes \cdots \otimes p_{t, x_{i_{n}}}\left(d y_{1}, \ldots, d y_{n}\right) \\
= & \int_{\Gamma_{X, 0}} G(\eta) \rho_{t, \gamma}(d \eta)
\end{aligned}
$$

where $\mathbf{x}=\left(x_{k}\right)_{k=1}^{\infty} \in I^{-1}\{\gamma\}$. The final inequality in the assertion immediately follows from (4.5) and (4.7). Hence, the measure $\rho_{t, \gamma}$ is from $\mathcal{M}_{\mathrm{lf}}\left(\Gamma_{X, 0}\right)$, and therefore, by Proposition 3.1, $\mathbf{P}_{t, \gamma} \in \mathcal{M}_{\mathrm{fm}}\left(\Gamma_{X}\right)$.

Remark 4.1. One could also start with a measure $\rho_{t, \gamma}$ on $\Gamma_{X, 0}$ that is given a priori by formula (4.7) for each $t>0$ and each $\gamma \in \Gamma_{X}$ satisfying (4.5), and then, using [18, Theorem 6.5], identify $\mathbf{P}_{t, \gamma}$ as the unique probability measure on $\Gamma_{X}$ whose correlation measure is $\rho_{t, \gamma}$.

Our next aim is to show that condition (4.5) is satisfied for $\pi_{m}$-a.a. $\gamma \in \Gamma_{X}$, at least under some additional conditions on the manifold $X$.

Let us assume that the manifold $X$ satisfies the following two conditions:

(C1) For each $t>0$, there exist constants $C_{t}>0$ and $\varepsilon_{t}>0$ such that

$$
p(t, x, y) \leq C_{t} \exp \left[-d(x, y)^{1+\varepsilon_{t}}\right], \quad t>0, x, y \in X .
$$

(C2) For some fixed $x_{0} \in X$,

$$
m\left(B\left(x_{0}, r\right)\right) \leq c_{x_{0}} r^{N}, \quad r>0,
$$

where $c_{x_{0}}>0, N \in \mathbb{N}$, and $B\left(x_{0}, r\right)$ denotes the geodesic ball with center at $x$ and radius $r$.

Concerning these conditions, in particular, the upper estimate of the heat kernel, we refer the reader e.g. to [10], [14], [15] and the references therein. For example, in the case of a manifold $X$ of nonnegative Ricci curvature, one has

$$
\begin{gathered}
p(t, x, y) \leq \frac{C}{m(B(y, \sqrt{t}))} \exp \left(-\frac{d(x, y)^{2}}{(4+\varepsilon) t}\right), \quad \varepsilon>0 \\
m(B(x, r)) \leq \text { const }_{d} r^{d}
\end{gathered}
$$

( $d$ being the dimension of $X)$. Thus, conditions $(\mathrm{C} 1)$ and $(\mathrm{C} 2)$ are satisfied if the manifold $X$ possesses the following additional property:

$$
\forall r>0: \quad \inf _{x \in X} m(B(x, r))>0,
$$


which is true, for example, in case of a manifold having bounded geometry (see [10]).

Now, we shall follow the idea of [21] to consider subsets of the configuration space on which some special functionals take finite values. So, for each $n \in \mathbb{N}$, we introduce the functional

$$
B_{n}(\gamma):=\left\langle\exp \left[-\frac{1}{n} d\left(x_{0}, \cdot\right)\right], \gamma\right\rangle=\sum_{x \in \gamma} \exp \left[-\frac{1}{n} d\left(x_{0}, x\right)\right], \quad \gamma \in \Gamma_{X},
$$

and define $\Gamma_{n} \in \mathcal{B}\left(\Gamma_{X}\right)$ by

$$
\Gamma_{n}:=\left\{\gamma \in \Gamma_{X}: B_{n}(\gamma)<\infty\right\} .
$$

Here, $x_{0}$ is as in (C2). Evidently, we have, for each $n \in \mathbb{N}, \Gamma_{n+1} \subset \Gamma_{n}$, and let

$$
\Gamma_{\infty}:=\bigcap_{n=1}^{\infty} \Gamma_{n}
$$

Let $d_{\mathrm{V}}$ be any metric on $\mathcal{M}(X)$ determining the vague topology. For example, we can take as $d_{\mathrm{V}}$ the metric $d_{\mathrm{K}}$ that was introduced in [32]:

$$
d_{\mathrm{K}}\left(\nu_{1}, \nu_{2}\right):=\sum_{i=1}^{\infty} 2^{-i} d_{\mathrm{K}, i}\left(\nu_{1}, \nu_{2}\right) /\left[1+d_{\mathrm{K}, i}\left(\nu_{1}, \nu_{2}\right)\right], \quad \nu_{1}, \nu_{2} \in \mathcal{M}(X),
$$

where

$$
\begin{aligned}
d_{\mathrm{K}, i}\left(\nu_{1}, \nu_{2}\right):=\sup \{\mid & \int_{X} f d\left(\nu_{1}-\nu_{2}\right) \mid: f: X \rightarrow \mathbb{R}, \\
& \left.\sup _{x, y \in X} \frac{d(f(x), f(y))}{d(x, y)} \leq 1, f(x)=0 \text { if } d\left(x_{0}, x\right) \geq i\right\} .
\end{aligned}
$$

The metric $d_{\mathrm{K}}$ is a generalization of the Kantorovich metric, and on any set of measures from $\mathcal{M}(X)$ which have uniformly bounded support, $d_{\mathrm{K}}$ is just equivalent to the Kantorovich metric.

Then, we can metrize the set $\Gamma_{\infty}$ as follows: for $\gamma_{1}, \gamma_{2} \in \Gamma_{\infty}$

$d_{\infty}\left(\gamma_{1}, \gamma_{2}\right):=d_{\mathrm{V}}\left(\gamma_{1}, \gamma_{2}\right)+\sum_{n=1}^{\infty} 2^{-n}\left|B_{n}\left(\gamma_{1}\right)-B_{n}\left(\gamma_{2}\right)\right| /\left[1+\left|B_{n}\left(\gamma_{1}\right)-B_{n}\left(\gamma_{2}\right)\right|\right]$.

Let $\mathcal{B}\left(\Gamma_{\infty}\right)$ denote the trace $\sigma$-algebra of $\mathcal{B}\left(\Gamma_{X}\right)$ on $\Gamma_{\infty}$. It can be shown that this $\sigma$-algebra coincides with the Borel $\sigma$-algebra on $\Gamma_{\infty}$ that corresponds to the topology generated by the $d_{\infty}$ metric. 
Proposition 4.2. Let $(\mathrm{C} 1)$ and $(\mathrm{C} 2)$ be satisfied. Then, $\Gamma_{\infty}$ is a set of full $\pi_{m}$ measure. Furthermore, for each $\gamma \in \Gamma_{\infty}$ condition (4.5) is satisfied and $\Gamma_{\infty}$ is a set of full $\mathbf{P}_{t, \gamma}$ measure for each $t>0$.

Proof. We have by (C2) that

$$
\begin{aligned}
\int_{\Gamma_{X}} B_{n}(\gamma) \pi_{m}(d \gamma) & =\int_{X} \exp \left[-\frac{1}{n} d\left(x_{0}, x\right)\right] m(d x) \\
& =\sum_{k=1}^{\infty} \int_{B\left(x_{0}, k\right) \backslash B\left(x_{0}, k-1\right)} \exp \left[-\frac{1}{n} d\left(x_{0}, x\right)\right] m(d x) \\
& \leq \sum_{k=1}^{\infty} \exp \left[-\frac{1}{n}(k-1)\right] m\left(B\left(x_{0}, k\right)\right) \\
& \leq \sum_{k=1}^{\infty} \exp \left[-\frac{1}{n}(k-1)\right] c_{x_{0}} k^{N}<\infty .
\end{aligned}
$$

Therefore, $B_{n}$ is $\pi_{m}$-a.e. finite, i.e., $\pi_{m}\left(\Gamma_{n}\right)=1$ for all $n \in \mathbb{N}$, which yields that $\pi_{m}\left(\Gamma_{\infty}\right)=1$.

Next, from (C1) we get, for each $r>0, t>0$, and $\gamma \in \Gamma_{\infty}$,

$$
\begin{aligned}
\sum_{x \in \gamma} p_{t, x}\left(B\left(x_{0}, r\right)\right) & =\sum_{x \in \gamma} \int_{B\left(x_{0}, r\right)} p(t, x, y) m(d y) \\
& \leq \widetilde{C}_{t} \sum_{x \in \gamma} \int_{B\left(x_{0}, r\right)} \exp [-d(x, y)] m(d y) \\
& \leq \widetilde{C}_{t} \sum_{x \in \gamma} \exp \left[-d\left(x_{0}, x\right)\right] \int_{B\left(x_{0}, r\right)} \exp \left[d\left(x_{0}, y\right)\right] m(d y)<\infty
\end{aligned}
$$

so that (4.5) is satisfied.

Finally, for each $\gamma \in \Gamma_{\infty}, t>0$, and $n \in \mathbb{N}$, we get from (C1), (C2) (cf. also (4.15)), and the monotone convergence theorem that

$$
\begin{aligned}
\int_{\Gamma_{X}} B_{n}(\xi) \mathbf{P}_{t, \gamma}(d \xi) & =\sum_{x \in \gamma} \int_{X} \exp \left[-\frac{1}{n} d\left(x_{0}, y\right)\right] p(t, x, y) m(d y) \\
& \leq \sum_{x \in \gamma} \int_{X} \exp \left[-\frac{1}{n} d\left(x_{0}, y\right)\right] C_{t} \exp \left[-d(x, y)^{1+\varepsilon_{t}}\right] m(d y) \\
& \leq C_{t, n} \sum_{x \in \gamma} \int_{X} \exp \left[-\frac{1}{n} d\left(x_{0}, y\right)\right] \exp \left[-\frac{1}{2 n} d(x, y)\right] m(d y)
\end{aligned}
$$




$$
\begin{aligned}
& \leq C_{t, n} \sum_{x \in \gamma} \exp \left[-\frac{1}{2 n} d\left(x_{0}, x\right)\right] \int_{X} \exp \left[-\frac{1}{2 n} d\left(x_{0}, y\right)\right] m(d y) \\
& <\infty
\end{aligned}
$$

which yields that $\mathbf{P}_{t, \gamma}\left(\Gamma_{\infty}\right)=1$.

Remark 4.2. Let $\pi_{z m}$ denote the Poisson measure on $\left(\Gamma_{X}, \mathcal{B}\left(\Gamma_{X}\right)\right)$ with intensity $z m$, where $z>0$. Since the correlation measure of $\pi_{z m}$ is the Lebesgue-Poisson measure $\lambda_{z m}$, it follows from the proof of Proposition 4.2 that $\pi_{z m}\left(\Gamma_{\infty}\right)=1$ for all $z>0$. Furthermore, let $\mu_{\nu, m}:=\int_{0}^{\infty} \pi_{z m} \nu(d z)$ be a mixed Poisson measure such that $\nu$ is a probability measure on $(0, \infty)$. Then, $\Gamma_{\infty}$ is a set of full $\mu_{\nu, m}$ measure.

\section{$\S 5$. Explicit Formula for the Heat Semigroup}

Due to Proposition 4.2, we can consider $\pi_{m}$ as a probability measure on $\left(\Gamma_{\infty}, \mathcal{B}\left(\Gamma_{\infty}\right)\right)$. In this section, we shall derive an explicit formula for the heat semigroup $\left(e^{-t H^{\Gamma}}\right)_{t \in \mathbb{R}_{+}}$.

Theorem 5.1. Let the conditions $(\mathrm{C} 1)$ and $(\mathrm{C} 2)$ be satisfied. Then, for each $F \in L^{2}\left(\Gamma_{\infty}, \pi_{m}\right)$, we have

$$
\left(e^{-t H^{\Gamma}} F\right)(\gamma)=\int_{\Gamma_{\infty}} F(\xi) \mathbf{P}_{t, \gamma}(d \xi)
$$

for $\pi_{m}-a . a . \gamma \in \Gamma_{\infty}$.

Proof. We start with the following

Lemma 5.1. Let $\widetilde{\mathcal{D}}_{1}$ denote the subset of $\mathcal{D}_{1}($ see $(2.9))$ given by

$$
\widetilde{\mathcal{D}}_{1}:=\{\varphi \in \mathcal{D} \mid \exists \delta \in(0,1):-\delta \leq \varphi \leq 0\} .
$$

Then, for any $\varphi \in \widetilde{\mathcal{D}}_{1}, \gamma \in \Gamma_{\infty}$, and $t>0$, we have

$$
\int_{\Gamma_{\infty}} \exp [\langle\log (1+\varphi), \xi\rangle] \mathbf{P}_{t, \gamma}(d \xi)=\exp \left[\left\langle\log \left(1+\int \varphi d p_{t, \bullet}\right), \gamma\right\rangle\right] .
$$

Proof. First, we observe that by Proposition 4.2

$$
\sum_{x \in \gamma} \int_{X}|\varphi(y)| p_{t, x}(d y)<\infty \quad \text { for each } \gamma \in \Gamma_{\infty} \text { and } \varphi \in \mathcal{D} .
$$


But for each $\gamma \in \Gamma_{\infty}, \mathbf{x}=\left(x_{k}\right)_{k=1}^{\infty} \in I^{-1}\{\gamma\}$, and $\varphi \in \widetilde{\mathcal{D}}_{1}$,

$$
\begin{aligned}
\int_{\Gamma_{\infty}} \exp [\langle\log (1+\varphi), \xi\rangle] \mathbf{P}_{t, \gamma}(d \xi) & =\prod_{k=1}^{\infty} \int_{X}(1+\varphi(y)) p_{t, x_{k}}(d y) \\
& =\exp \left[\left\langle\log \left(1+\int \varphi d p_{t, \bullet}\right), \gamma\right\rangle\right]
\end{aligned}
$$

Lemma 5.2. For any measurable function $F: \Gamma_{\infty} \rightarrow \mathbb{R}_{+}$, we have

$$
\int_{\Gamma_{\infty}} \int_{\Gamma_{\infty}} F(\xi) \mathbf{P}_{t, \gamma}(d \xi) \pi_{m}(d \gamma)=\int_{\Gamma_{\infty}} F(\gamma) \pi_{m}(d \gamma) .
$$

Proof. It is easy to check that $\left\{\exp [\langle\log (1+\varphi), \cdot\rangle] \mid \varphi \in \widetilde{\mathcal{D}}_{1}\right\}$ is stable under multiplication and that it contains a countable subset separating the points of $\Gamma_{\infty}$, so it generates $\mathcal{B}\left(\Gamma_{\infty}\right)$. Therefore, we only have to check (5.2) for $F:=\exp [\langle\log (1+\varphi), \cdot\rangle], \varphi \in \widetilde{\mathcal{D}}_{1}$. But for such functions (5.2) immediately follows from Lemma 5.1. Indeed, (2.4) extends to all functions $\varphi: X \rightarrow \mathbb{R}_{+}$ which are increasing limits of functions $\varphi_{n} \in \mathcal{D}, n \in \mathbb{N}$, such as $\log \left(1+\int \varphi d p_{t, \bullet}\right)$. Furthermore, $\iint \varphi d p_{t, \bullet} d m=\int \varphi d m$, since $H^{X}$ is assumed to be conservative.

Now, we can easily finish the proof of the theorem. It follows from Lemma 5.2 that, if $A \in \mathcal{B}\left(\Gamma_{\infty}\right)$ is of zero $\pi_{m}$ measure, then $\mathbf{P}_{t, \gamma}(A)=0$ for $\pi_{m}$-a.e. $\gamma \in \Gamma_{\infty}$. Moreover, using the Cauchy-Schwarz inequality and Lemma 5.2, we get

$$
\begin{aligned}
\int_{\Gamma_{\infty}}\left(\int_{\Gamma_{\infty}} F(\xi) \mathbf{P}_{t, \gamma}(d \xi)\right)^{2} \pi_{m}(d \gamma) & \leq \int_{\Gamma_{\infty}} \int_{\Gamma_{\infty}}|F(\xi)|^{2} \mathbf{P}_{t, \gamma}(d \xi) \pi_{m}(d \gamma) \\
& =\int_{\Gamma_{\infty}}|F(\gamma)|^{2} \pi_{m}(d \gamma)
\end{aligned}
$$

Thus, for each $t>0$, we can define a linear continuous operator

$$
\mathbf{P}_{t}: L^{2}\left(\Gamma_{\infty}, \pi_{m}\right) \rightarrow L^{2}\left(\Gamma_{\infty}, \pi_{m}\right)
$$

by setting

$$
\left(\mathbf{P}_{t} F\right)(\gamma):=\int_{\Gamma_{\infty}} F(\xi) \mathbf{P}_{t, \gamma}(d \xi)
$$

By Proposition 2.1 and Lemma 5.1, the action of the operator $\mathbf{P}_{t}$ coincides with the action of the operator $e^{-t H^{\Gamma}}$ on the $\operatorname{set}\left\{\exp [\langle\log (1+\varphi), \cdot\rangle] \mid \varphi \in \widetilde{\mathcal{D}}_{1}\right\}$, 
which is total in $L^{2}\left(\Gamma_{\infty}, \pi_{m}\right)$ (i.e., its linear hull is a dense set in $L^{2}\left(\Gamma_{\infty}, \pi_{m}\right)$ ). Hence, we get the equality $e^{-t H^{\Gamma}}=\mathbf{P}_{t}$, which proves the theorem.

In what follows, for a measurable function $F$ on $\Gamma_{\infty}$, we set

$$
\left(\mathbf{P}_{t} F\right)(\gamma):=\int_{\Gamma_{\infty}} F(\xi) \mathbf{P}_{t, \gamma}(d \xi), \quad t>0, \gamma \in \Gamma_{\infty}
$$

provided the integral on the right hand side exists. Hence, by virtue of Theorem 5.1, $\mathbf{P}_{t} F$ is a $\pi_{m}$-version of $e^{-t H^{\Gamma}} F$ for each $F \in L^{2}\left(\Gamma_{\infty}, \pi_{m}\right)$.

Remark 5.1. One can easily prove an explicit formula for the heat semigroup $\left(e^{-t H^{\Gamma}}\right)_{t \in \mathbb{R}_{+}}$in the weak sense. More specifically, we define for each $t>0$ a function $R_{t}: \Gamma_{X, 0} \times \Gamma_{X, 0} \rightarrow \mathbb{R}$ setting: $R_{t}(\eta, \theta):=0$ if $|\eta| \neq|\theta|$, $R_{t}(\{\varnothing\},\{\varnothing\})=1$, and for $\eta=\left\{x_{1}, \ldots, x_{n}\right\}, \theta=\left\{y_{1}, \ldots, y_{n}\right\}, n \in \mathbb{N}$,

$$
R_{t}(\eta, \theta):=\sum_{\sigma \in S_{n}} \prod_{k=1}^{n} p_{t}\left(x_{k}, y_{\sigma(k)}\right)
$$

where $p_{t}(x, y):=p(t, x, y)$. Suppose that conditions $(\mathrm{C} 1)$ and $(\mathrm{C} 2)$ are satisfied. Then, for arbitrary measurable functions $G_{1}, G_{2}: \Gamma_{X, 0} \rightarrow \mathbb{R}$ such that

$$
\int_{\Gamma_{X, 0}} \int_{\Gamma_{X}, 0}\left|G_{1}(\theta)\right| \cdot\left[R_{t}(\eta, \theta) \star_{\eta}\left|G_{2}(\eta)\right|\right] \lambda_{m}(d \eta) \lambda_{m}(d \theta)<\infty \quad \text { for all } t>0
$$

( $\star_{\eta}$ denoting the $\star$-convolution with respect to the $\eta$ variable), we have

$$
\int_{\Gamma_{\infty}}\left|\mathbf{P}_{t} F_{1}(\gamma)\right|\left|F_{2}(\gamma)\right| \pi_{m}(d \gamma)<\infty
$$

where $F_{1}(\gamma)=\left(K G_{1}\right)(\gamma), F_{2}(\gamma)=\left(K G_{2}\right)(\gamma)$, and

$$
\begin{aligned}
\int_{\Gamma_{\infty}} & \left(\mathbf{P}_{t} F_{1}\right)(\gamma) F_{2}(\gamma) \pi_{m}(d \gamma) \\
\quad= & \int_{\Gamma_{X, 0}} \int_{\Gamma_{X, 0}} G_{1}(\theta) \cdot\left[R_{t}(\eta, \theta) \star_{\eta} G_{2}(\eta)\right] \lambda_{m}(d \eta) \lambda_{m}(d \theta) .
\end{aligned}
$$

Now, we define a family of probability kernels $\left(\mathbf{P}_{t}\right)_{t \in \mathbb{R}_{+}}$on the space $\left(\Gamma_{\infty}, \mathcal{B}\left(\Gamma_{\infty}\right)\right)$ setting

$$
\mathbf{P}_{t}(\gamma, A):=\mathbf{P}_{t, \gamma}(A), \quad \gamma \in \Gamma_{\infty}, A \in \mathcal{B}\left(\Gamma_{\infty}\right), t \in \mathbb{R}_{+},
$$

where

$$
\mathbf{P}_{0, \gamma}:=\varepsilon_{\gamma}
$$


Since $\gamma \mapsto \mathbf{P}_{t} F(\gamma)$ is measurable for $F$ in the linear span of $\{\exp [\langle\log (1+$ $\left.\varphi), \cdot\rangle] \mid \varphi \in \widetilde{\mathcal{D}}_{1}\right\}$ by Lemma 5.1, a monotone class argument shows that, indeed, $\gamma \mapsto \mathbf{P}_{t}(\gamma, A)$ is $\mathcal{B}\left(\Gamma_{\infty}\right)$-measurable for all $A \in \mathcal{B}\left(\Gamma_{\infty}\right)$.

We finish this section with the following proposition.

Proposition 5.1. Let $(\mathrm{C} 1)$ and $(\mathrm{C} 2)$ be satisfied. Then, $\left(\mathbf{P}_{t}\right)_{t \in \mathbb{R}_{+}}$is a Markov semigroup of kernels on $\left(\Gamma_{\infty}, \mathcal{B}\left(\Gamma_{\infty}\right)\right)$.

Proof. The Markov property of the kernels $\mathbf{P}_{t}$, i.e., $\mathbf{P}_{t}\left(\gamma, \Gamma_{\infty}\right)=1, \gamma \in$ $\Gamma_{\infty}$, follows from Proposition 4.2.

Let us show the semigroup property: $\mathbf{P}_{t} \mathbf{P}_{s}=\mathbf{P}_{t+s}, t, s \in \mathbb{R}_{+}$. To this end, we fix $t, s>0, \gamma \in \Gamma_{\infty}$, and $A \in \mathcal{B}\left(\Gamma_{\infty}\right)$. Then, by the construction of the measure $\mathbf{P}_{t, \gamma}$ and the semigroup property of the heat kernel on $X$, we get

$$
\begin{aligned}
\left(\mathbf{P}_{t} \mathbf{P}_{s}\right)(\gamma, A) & =\int_{\Gamma_{\infty}} \mathbf{P}_{s, \xi}(A) \mathbf{P}_{t, \gamma}(d \xi)=\int_{\widetilde{X}^{\mathbb{N}}} \mathbf{P}_{s, I \mathbf{y}}(A) \mathbb{P}_{t, \mathbf{x}}(d \mathbf{y}) \\
& =\int_{\widetilde{X}^{\mathbb{N}}} \mathbb{P}_{s, \mathbf{y}}\left(I^{-1} A\right) \mathbb{P}_{t, \mathbf{x}}(d \mathbf{y})=\int_{X^{\mathbb{N}}} \mathbb{P}_{s, \mathbf{y}}\left(I^{-1} A\right) \mathbb{P}_{t, \mathbf{x}}(d \mathbf{y}) \\
& =\mathbb{P}_{t+s, \mathbf{x}}\left(I^{-1} A\right)=\mathbf{P}_{t+s, \gamma}(A)=\mathbf{P}_{t+s}(\gamma, A),
\end{aligned}
$$

where $\mathbf{x} \in I^{-1}\{\gamma\}$.

\section{§6. A Strong Feller Property of the Heat Semigroup}

Let us introduce a new metric $d_{1}$ on the set $\Gamma_{\infty}$ as follows:

$$
d_{1}\left(\gamma_{1}, \gamma_{2}\right):=d_{\mathrm{V}}\left(\gamma_{1}, \gamma_{2}\right)+\left|B_{1}\left(\gamma_{1}\right)-B_{1}\left(\gamma_{2}\right)\right|, \quad \gamma_{1}, \gamma_{2} \in \Gamma_{\infty}
$$

Evidently, convergence with respect to the $d_{\infty}$ metric implies convergence with respect to the $d_{1}$ metric.

In this section, we shall show that the "concrete version" $\left(\mathbf{P}_{t}\right)_{t \in \mathbb{R}_{+}}$of the heat semigroup $\left(e^{-t H^{\Gamma}}\right)_{t \in \mathbb{R}_{+}}$constructed in the previous section possesses a kind of strong Feller property with respect to the metric $d_{1}$, and therefore also with respect to $d_{\infty}$.

Theorem 6.1. Let $(\mathrm{C} 1)$ and $(\mathrm{C} 2)$ hold. Let $G: \Gamma_{X, 0} \rightarrow \mathbb{R}$ be a measurable function satisfying the following condition:

$$
\forall c>0: \quad \int_{\Gamma_{X, 0}}|G(\eta)| \lambda_{m_{c}}(d \eta)<\infty,
$$


where, for each $c>0, \lambda_{m_{c}}$ is the Lebesgue-Poisson measure on $\Gamma_{X, 0}$ with intensity

$$
m_{c}(d x):=c e^{d\left(x_{0}, x\right)} m(d x)
$$

Then, for each $t>0$, the function

$$
\Gamma_{\infty} \ni \gamma \mapsto\left(\mathbf{P}_{t}(K G)\right)(\gamma)=\int_{\Gamma_{\infty}}(K G)(\xi) \mathbf{P}_{t, \gamma}(d \xi) \in \mathbb{R}
$$

is continuous with respect to the metric $d_{1}$.

Proof. Let $\gamma \in \Gamma_{\infty}$. By Propositions 4.1, 4.2 and the definition of correlation functions, we see that for each $t>0$ the measure $\rho_{t, \gamma}$ is absolutely continuous with respect to the Lebesgue-Poisson measure $\lambda_{m}$, and the correlation functions $k_{t, \gamma}^{(n)}$ of $\mathbf{P}_{t, \gamma}$ are given by

$$
k_{t, \gamma}^{(n)}(\theta)=\sum_{\left(i_{1}, \ldots, i_{n}\right) \in \widetilde{\mathbb{N}}^{n}} \prod_{k=1}^{n} p_{t}\left(x_{i_{k}}, y_{k}\right) \quad \text { for } \gamma=\left\{x_{i}\right\}_{i=1}^{\infty} \text { and } \theta=\left\{y_{1}, \ldots, y_{n}\right\}
$$

where

$$
\widetilde{\mathbb{N}}^{n}:=\left\{\left(i_{i}, \ldots, i_{n}\right) \in \mathbb{N}^{n}: i_{k} \neq i_{l} \text { if } k \neq l\right\}
$$

Denote

$$
k_{t}(\gamma, \theta):=k_{t, \gamma}(\theta):=\frac{d \rho_{t, \gamma}}{d \lambda_{m}}(\theta)
$$

so that $k_{t, \gamma}(\theta)=k_{t, \gamma}^{(n)}(\theta)$ for $|\theta|=n$.

By using (C1), we get

$$
\begin{aligned}
& \left|k_{t}\left(\gamma,\left\{y_{1}, \ldots, y_{n}\right\}\right)\right| \\
& \quad \leq \prod_{k=1}^{n}\left(\sum_{x \in \gamma} p_{t}\left(x, y_{k}\right)\right) \\
& \quad \leq \prod_{k=1}^{n}\left(\sum_{x \in \gamma} C_{t}^{\prime} \exp \left[-d\left(x, y_{k}\right)\right]\right) \\
& \quad \leq\left(C_{t}^{\prime} \sum_{x \in \gamma} \exp \left[-d\left(x_{0}, x\right)\right]\right)^{n} \exp \left[d\left(x_{0}, y_{1}\right)+\cdots+d\left(x_{0}, y_{n}\right)\right] .
\end{aligned}
$$


Hence, (6.1) and (6.2) imply that $G \in L^{1}\left(\Gamma_{X, 0}, \mathcal{B}\left(\Gamma_{X, 0}\right), \rho_{t, \gamma}\right)$. Therefore, if $\gamma_{j} \rightarrow \gamma$ in $\Gamma_{\infty}$ with respect to $d_{1}$, by Proposition 3.2 and (6.5) we have to prove that

$$
\int_{\Gamma_{X}, 0} G(\eta) k_{t}\left(\gamma^{j}, \eta\right) \lambda_{m}(d \eta) \rightarrow \int_{\Gamma_{X}, 0} G(\eta) k_{t}(\gamma, \eta) \lambda_{m}(d \eta) \quad \text { as } j \rightarrow \infty .
$$

First, we show that

$$
k_{t}\left(\gamma^{j}, \eta\right) \rightarrow k_{t}(\gamma, \eta) \quad \text { as } j \rightarrow \infty \text { for each fixed } \eta \in \Gamma_{X, 0} .
$$

Since $\gamma^{j} \rightarrow \gamma$ in the $d_{1}$ metric, we have, particularly, that $\gamma^{j} \rightarrow \gamma$ in the $d_{\mathrm{V}}$ metric. We claim that there exists a numeration of the points of the configurations $\gamma^{j}, j \in \mathbb{N}$, and of $\gamma$ such that

$$
\text { (6.9) } \gamma^{j}=\left\{x_{k}^{j}\right\}_{k=1}^{\infty}, \quad \gamma=\left\{x_{k}\right\}_{k=1}^{\infty}, \quad \forall k \in \mathbb{N}: \quad d\left(x_{k}^{j}, x_{k}\right) \rightarrow 0 \text { as } j \rightarrow \infty .
$$

Indeed, let us fix any numeration of points of $\gamma$ such that

$$
\gamma=\left\{x_{k}\right\}_{k=1}^{\infty}, \quad d\left(x_{0}, x_{k+1}\right) \geq d\left(x_{0}, x_{k}\right), \quad k \in \mathbb{N} .
$$

Next, we fix positive numbers $r_{n}, n \in \mathbb{N}$, so that

$$
\begin{gathered}
r_{n+1}>r_{n}, \quad n \in \mathbb{N}, \quad r_{n} \rightarrow \infty \text { as } n \rightarrow \infty, \\
\forall x \in\left(\bigcup_{j=1}^{\infty} \gamma^{j}\right) \cup \gamma: \quad d\left(x_{0}, x\right) \neq r_{n}, \\
k_{1}:=\left|\gamma \cap B\left(x_{0}, r_{1}\right)\right|>0, \quad k_{n}:=\left|\gamma \cap\left(B\left(x_{0}, r_{n}\right) \backslash B\left(x_{0}, r_{n-1}\right)\right)\right|>0, \quad n \geq 2 .
\end{gathered}
$$

Since $\gamma_{j} \rightarrow \gamma$ vaguely, we then conclude that there exist $j_{1} \in \mathbb{N}$ such that

$$
\left|\gamma^{j} \cap B\left(x_{0}, r_{1}\right)\right|=k_{1} \quad \text { for all } j \geq j_{1},
$$

and a numeration of the points of $\gamma^{j} \cap B\left(x_{0}, r_{1}\right), j \geq j_{1}$, such that

$$
\gamma^{j} \cap B\left(x_{0}, r_{1}\right)=\left\{x_{k}^{j}\right\}_{k=1}^{k_{1}}, \quad x_{k}^{j} \rightarrow x_{k} \text { as } j \rightarrow \infty \text { for all } k=1, \ldots, k_{1} .
$$

Next, there exist $j_{2} \in \mathbb{N}, j_{2}>j_{1}$, such that

$$
\left|\gamma^{j} \cap\left(B\left(x_{0}, r_{2}\right) \backslash B\left(x_{0}, r_{1}\right)\right)\right|=k_{2} \quad \text { for all } j \geq j_{2},
$$

and a numeration of the points of $\gamma^{j} \cap\left(B\left(x_{0}, r_{2}\right) \backslash B\left(x_{0}, r_{1}\right)\right), j \geq j_{2}$, such that

$$
\gamma^{j} \cap\left(B\left(x_{0}, r_{2}\right) \backslash B\left(x_{0}, r_{1}\right)\right)=\left\{x_{k}^{j}\right\}_{k=k_{1}+1}^{k_{1}+k_{2}},
$$




$$
x_{k}^{j} \rightarrow x_{k} \text { as } j \rightarrow \infty \text { for all } k=k_{1}+1, \ldots, k_{1}+k_{2} .
$$

Continuing this procedure by induction, we get for each $j_{n} \leq j<j_{n+1}$ a numeration $\left\{x_{k}^{j}\right\}_{k=1}^{k_{1}+\cdots+k_{n}}$ of the points of $\gamma^{j} \cap B\left(x_{0}, r_{n}\right)$. For such $j$ we choose an arbitrary numeration $\left\{x_{k}^{j}\right\}_{k=k_{1}+\cdots+k_{n}+1}^{\infty}$ of $\gamma^{j} \cap B\left(x_{0}, r_{n}\right)^{\mathrm{c}}$, and therefore obtain a numeration $\left\{x_{k}^{j}\right\}_{k=1}^{\infty}$ of $\gamma_{j}$. Since $j_{n} \rightarrow \infty$, we thus have a numeration of all $\gamma^{j}$ with $j \geq j_{1}$ (for the first $j_{1}-1$ configurations, we again take an arbitrary numeration). Now, for any fixed $l \in \mathbb{N}$, take the minimal $n(l) \in \mathbb{N}$ satisfying $x_{l} \in B\left(x_{0}, r_{n(l)}\right)$ (this $n(l)$ always exists since $r_{n} \rightarrow \infty$ ). Then, $k_{1}+\cdots+k_{n(l)-1}+1 \leq l \leq k_{1}+\cdots+k_{n(l)}$ (where $k_{1}+\cdots+k_{n(l)-1}:=0$ if $n(l)=1)$. By induction, the sequence $\left(x_{k}^{j}\right)_{j=j_{n(l)}}^{\infty}$ converges to $x_{k}$ as $j \rightarrow \infty$ for each $k$ satisfying $k_{1}+\cdots+k_{n(l)-1}+1 \leq k \leq k_{1}+\cdots+k_{n(l)}$, which immediately yields that the sequence $\left(x_{l}^{j}\right)_{j=1}^{\infty}$ converges to $x_{l}$ as $j \rightarrow \infty$.

According to (6.4), (6.8) is, therefore, equivalent to the convergence

$$
\sum_{\left(i_{1}, \ldots, i_{n}\right) \in \widetilde{\mathbb{N}}^{n}} \prod_{k=1}^{n} p_{t}\left(x_{i_{k}}^{j}, y_{k}\right) \rightarrow \sum_{\left(i_{1}, \ldots, i_{n}\right) \in \widetilde{\mathbb{N}}^{n}} \prod_{k=1}^{n} p_{t}\left(x_{i_{k}}, y_{k}\right) \quad \text { as } j \rightarrow \infty
$$

for each fixed $\left(y_{1}, \ldots, y_{n}\right) \in \widetilde{X}^{n}, n \in \mathbb{N}$.

We now claim that, for any fixed $\varepsilon>0$, there exist $J, K \in \mathbb{N}$ such that

$$
\forall j>J: \quad \sum_{k=K+1}^{\infty} \exp \left[-d\left(x_{0}, x_{k}^{j}\right)\right]<\varepsilon, \quad \sum_{k=K+1}^{\infty} \exp \left[-d\left(x_{0}, x_{k}\right)\right]<\varepsilon .
$$

Indeed, choose any $K \in \mathbb{N}$ such that

$$
\sum_{k=K+1}^{\infty} \exp \left[-d\left(x_{0}, x_{k}\right)\right]<\frac{\varepsilon}{3},
$$

then choose any $J_{1} \in \mathbb{N}$ such that

$$
\forall j>J_{1}: \quad\left|\sum_{k=1}^{K} \exp \left[-d\left(x_{0}, x_{k}^{j}\right)\right]-\sum_{k=1}^{K} \exp \left[-d\left(x_{0}, x_{k}\right)\right]\right|<\frac{\varepsilon}{3},
$$

and finally take any $J_{2} \in \mathbb{N}$ such that

$$
\forall j>J_{2}: \quad\left|\sum_{k=1}^{\infty} \exp \left[-d\left(x_{0}, x_{k}^{j}\right)\right]-\sum_{k=1}^{\infty} \exp \left[-d\left(x_{0}, x_{k}\right)\right]\right|<\frac{\varepsilon}{3} .
$$

Then, it follows from (6.12)-(6.14) that (6.11) holds with $K$ as above and $J:=\max \left\{J_{1}, J_{2}\right\}$. 
Now, we conclude from (C1) that, for each fixed $t>0$ and $y \in X$, there exists const $_{t, y}>0$ such that

$$
p_{t}(x, y) \leq \text { const }_{t, y} \exp \left[-d\left(x_{0}, x\right)\right]
$$

Thus, from (6.9), (6.11) and (6.15), we easily derive that

$$
\sum_{k=1}^{\infty}\left|p_{t}\left(x_{k}^{j}, y\right)-p_{t}\left(x_{k}, y\right)\right| \rightarrow 0 \quad \text { as } j \rightarrow \infty .
$$

Thus, (6.10) holds for $n=1$.

Next, we show that (6.10) holds for $n=2$, i.e.,

$$
\begin{aligned}
& \sum_{i_{1}=1}^{\infty} \mid p_{t}\left(x_{i_{1}}^{j}, y_{1}\right) \sum_{i_{2} \in \mathbb{N}, i_{2} \neq i_{1}} p_{t}\left(x_{i_{2}}^{j}, y_{2}\right) \\
& \quad-p_{t}\left(x_{i_{1}}, y_{1}\right) \sum_{i_{2} \in \mathbb{N}, i_{2} \neq i_{1}} p_{t}\left(x_{i_{2}}, y_{2}\right) \mid \rightarrow 0 \quad \text { as } j \rightarrow \infty .
\end{aligned}
$$

It follows from (6.9) and (6.16) that, for each $i_{1} \in \mathbb{N}$,

$$
p_{t}\left(x_{i_{1}}^{j}, y_{1}\right) \sum_{i_{2} \in \mathbb{N}, i_{2} \neq i_{1}} p_{t}\left(x_{i_{2}}^{j}, y_{2}\right) \rightarrow p_{t}\left(x_{i_{1}}, y_{1}\right) \sum_{i_{2} \in \mathbb{N}, i_{2} \neq i_{1}} p_{t}\left(x_{i_{2}}, y_{2}\right) \quad \text { as } j \rightarrow \infty \text {. }
$$

Moreover, we get from (6.16) that

$$
\sum_{i_{2} \in \mathbb{N}, i_{2} \neq i_{1}} p_{t}\left(x_{i_{2}}^{j}, y_{2}\right) \leq \sum_{i_{2}=1}^{\infty} p_{t}\left(x_{i_{2}}^{j}, y_{2}\right) \leq \text { const } \quad \forall j \in \mathbb{N} .
$$

Thus, we obtain (6.17) from (6.11), (6.15), (6.18) and (6.19).

Continuing this way, by induction we prove (6.10) for each $n \in \mathbb{N}$.

By virtue of the majorized convergence theorem, it still remains to verify that all the functions $G(\cdot) k_{t}\left(\gamma^{j}, \cdot\right)$ are majorized by a function from $L^{1}\left(\lambda_{m}\right)$. But it follows from (6.6) that

$$
\left|k_{t}\left(\gamma^{j},\left\{y_{1}, \ldots, y_{n}\right\}\right)\right| \leq \mathrm{const}_{t}^{n} \exp \left[d\left(x_{0}, y_{1}\right)+\cdots+d\left(x_{0}, y_{n}\right)\right]
$$

Thus, (6.1) implies the assertion of the theorem.

We have also the following theorem. 
Theorem 6.2. Let $\mathbb{D}$ denote the set of all measurable functions $G=$ $\left(G^{(n)}\right)_{n=0}^{\infty}$ on $\Gamma_{X, 0}$ such that there exist $\varepsilon=\varepsilon(G), C=C(G)>0$ such that

$$
\begin{aligned}
\left|G^{(n)} \circ T_{n}\left(x_{1}, \ldots, x_{n}\right)\right| \leq & C^{n} \exp \left[-(1+\varepsilon)\left(d\left(x_{0}, x_{1}\right)+\cdots+d\left(x_{0}, x_{n}\right)\right)\right], \\
& \left(x_{1}, \ldots, x_{n}\right) \in \widetilde{X}^{n}, n \in \mathbb{N} .
\end{aligned}
$$

We define

$$
\mathbf{D}:=\left\{(K G)\left|\Gamma_{\infty}\right| G \in \mathbb{D}\right\}
$$

Then,

$$
\forall p \geq 1: \quad \mathbf{D} \subset L^{p}\left(\Gamma_{\infty}, \pi_{m}\right)
$$

and

$$
\forall t>0: \quad \mathbf{P}_{t} \mathbf{D} \subset \mathbf{D} .
$$

Furthermore, for each $F \in \mathbf{D}, \mathbf{P}_{t} F$ is a continuous function on $\Gamma_{\infty}$ with respect to the metric $d_{1}$.

Proof. By virtue of (4.15), (6.20) implies (6.1), and hence by Theorem 6.1, $\mathbf{P}_{t} F$ is a continuous function on $\Gamma_{\infty}$ with respect to $d_{1}$ for each $F \in \mathbf{D}$.

Next, $\mathbb{D} \subset L^{1}\left(\Gamma_{X, 0}, \lambda_{m}\right)$, and therefore, by Proposition 3.2,

$$
\mathbf{D} \subset L^{1}\left(\Gamma_{\infty}, \pi_{m}\right) .
$$

For each $\eta \in \Gamma_{X, 0}, \sum_{\theta \subset \eta} 1=2^{|\eta|}$, which yields that

$$
\sum_{\left(\theta_{1}, \theta_{2}, \theta_{3}\right) \in \mathcal{P}_{3}(\eta)} 1 \leq 6 \cdot 4^{|\eta|} .
$$

By (6.24) and definition (3.5), we get that $G_{1} \star G_{2} \in \mathbb{D}$ for arbitrary $G_{1}, G_{2} \in \mathbb{D}$. Consequently, by (3.6),

$$
F_{1} \cdot F_{2} \in \mathbf{D}, \quad F_{1}, F_{2} \in \mathbf{D} .
$$

From (6.23) and (6.25), we get (6.21).

Finally, let us show that the set $\mathbf{D}$ is invariant under the action of $\mathbf{P}_{t}$. By (4.7) and (4.8), we have

$$
\left(\mathbf{P}_{t}(K G)\right)(\gamma)=\left(K\left(\widetilde{\mathbf{P}}_{t} G\right)\right)(\gamma), \quad \gamma \in \Gamma_{\infty}, G \in \mathbb{D}
$$


where $\widetilde{\mathbf{P}}_{t} G=\left(\left(\widetilde{\mathbf{P}}_{t} G\right)^{(n)}\right)_{n=0}^{\infty}$ is given by

$$
\begin{gathered}
\left(\widetilde{\mathbf{P}}_{t} G\right)^{(0)}=G^{(0)}, \\
\left(\widetilde{\mathbf{P}}_{t} G\right)^{(n)} \circ T_{n}\left(x_{1}, \ldots, x_{n}\right)=\int_{\widetilde{X}^{n}} G^{(n)} \circ T_{n}\left(y_{1}, \ldots, y_{n}\right) p_{t, x_{1}}\left(d y_{1}\right) \cdots p_{t, x_{n}}\left(d y_{n}\right), \\
\left(x_{1}, \ldots, x_{n}\right) \in \widetilde{X}^{n}, n \in \mathbb{N} .
\end{gathered}
$$

Now, by using (C1), we derive, for $G=\left(G^{(n)}\right)_{n=0}^{\infty}$ satisfying (6.20) with some $\varepsilon>0$ and $C>0$,

$$
\begin{aligned}
& \left|\left(\widetilde{\mathbf{P}}_{t} G\right)^{(n)} \circ T_{n}\left(x_{1}, \ldots, x_{n}\right)\right| \\
& \quad \leq \int_{X^{n}} C^{n} \exp \left[-(1+\varepsilon)\left(d\left(x_{0}, y_{1}\right)+\cdots+d\left(x_{0}, y_{n}\right)\right)\right] \\
& \quad \times\left(C_{t}^{\prime}\right)^{n} \exp \left[-\left(1+\frac{\varepsilon}{2}\right)\left(d\left(x_{1}, y_{1}\right)+\cdots+d\left(x_{n}, y_{n}\right)\right)\right] m\left(d y_{1}\right) \cdots m\left(d y_{n}\right) \\
& \leq\left(C C_{t}^{\prime}\right)^{n} \exp \left[-\left(1+\frac{\varepsilon}{2}\right)\left(d\left(x_{0}, x_{1}\right)+\cdots+d\left(x_{0}, x_{n}\right)\right)\right] \\
& \quad \times\left(\int_{X} \exp \left[-\frac{\varepsilon}{2} d\left(x_{0}, y\right)\right] m(d y)\right)^{n} .
\end{aligned}
$$

Hence, because of (4.15), $\widetilde{\mathbf{P}}_{t} G \in \mathbb{D}$, and (6.22) follows from (6.26).

For an arbitrary measurable, bounded, symmetric function $G^{(n)}\left(x_{1}\right.$, $\left.\ldots, x_{n}\right)$ on $X^{n}, n \in \mathbb{N}$, with bounded support, one can introduce the following monomial on $\Gamma_{\infty}$ of $n$-th order with kernel $G^{(n)}$ :

$$
\Gamma_{\infty} \ni \gamma \mapsto\left\langle G^{(n)},: \gamma^{\otimes n}:\right\rangle:=\sum_{\left\{x_{1}, \ldots, x_{n}\right\} \subset \gamma} G^{(n)}\left(x_{1}, \ldots, x_{n}\right)=\left(K G^{(n)}\right)(\gamma) .
$$

It is natural to call a finite sum of functions of such type and a constant a cylindrical polynomial on $\Gamma_{\infty}$ with bounded coefficients. We denote by $\mathcal{F} \mathcal{P}_{\text {bc }}\left(\Gamma_{\infty}\right)$ the set of all such polynomials on $\Gamma_{\infty}$. Thus, $\mathcal{F} \mathcal{P}_{\mathrm{bc}}\left(\Gamma_{X}\right)$ is nothing but the image of the set $B_{\mathrm{bs}}\left(\Gamma_{X, 0}\right)$ under the $K$-transform.

Since every function $G \in B_{\mathrm{bs}}\left(\Gamma_{X, 0}\right)$ satisfies (6.20), we get the following consequence of Theorem 6.2:

Corollary 6.1. We have the inclusion $\mathcal{F P}_{\mathrm{bc}}\left(\Gamma_{\infty}\right) \subset \mathbf{D}$. In particular, for each polynomial $F \in \mathcal{F} \mathcal{P}_{\mathrm{bc}}\left(\Gamma_{\infty}\right)$, the function $\mathbf{P}_{t} F$ is continuous on $\Gamma_{\infty}$ with respect to the metric $d_{1}$.

A measurable function $F: \Gamma_{\infty} \rightarrow \mathbb{R}$ is called local if there exist an open, relatively compact set $\Lambda \subset X$ and a measurable function $\widetilde{F}: \Gamma_{\Lambda, 0} \rightarrow \mathbb{R}$ such 
that $F(\gamma)=\widetilde{F}\left(\gamma_{\Lambda}\right)$ for all $\gamma \in \Gamma_{\infty}$. The following corollary is an analog of the classical strong Feller property for the heat semigroup on the configuration space.

Corollary 6.2. $\quad$ Each measurable bounded local function $F: \Gamma_{\infty} \rightarrow \mathbb{R}$ belongs to $\mathbf{D}$, and hence the function $\mathbf{P}_{t} F$ is continuous on $\Gamma_{\infty}$ with respect to the metric $d_{1}$.

Proof. Let $F(\gamma)=\widetilde{F}\left(\gamma_{\Lambda}\right)$ with $\Lambda$ and $\widetilde{F}$ as above. By [18, Proposition 3.5], one can explicitly calculate the inverse $K$-transform of $F$ :

$$
\left(K^{-1} F\right)(\eta)= \begin{cases}\sum_{\theta \subset \eta}(-1)^{|\eta \backslash \theta|} \widetilde{F}(\theta), & \text { if } \eta \in \Gamma_{\Lambda, 0} \\ 0, & \text { otherwise. }\end{cases}
$$

Set $G=\left(G^{(n)}\right)_{n=0}^{\infty}:=K^{-1} F$. Let $C:=\sup |F|$. Since $\sum_{\theta \subset \eta} 1=2^{|\eta|}$, we conclude from (6.27) that, for each $n \in \mathbb{N},\left\{G^{(n)} \neq 0\right\} \subset \Gamma_{\Lambda}^{(n)}$ and $\left|G^{(n)}\right|$ is bounded by the constant $C 2^{n}$. Therefore, $G \in \mathbb{D}$ and $F=K G \in \mathbf{D}$.

Remark 6.1. Let $t>0$. As a consequence of Corollary 6.2 we have that any Markovian kernel $\widetilde{\mathbf{P}}_{t}$ on $\left(\Gamma_{\infty}, \mathcal{B}\left(\Gamma_{\infty}\right)\right)$ such that $\widetilde{\mathbf{P}}_{t} F$ is continuous with respect to $d_{1}$ and $\widetilde{\mathbf{P}}_{t} F$ is a $\pi_{m}$-version of $e^{-t H^{\Gamma}} F$ for each measurable bounded local function $F: \Gamma_{\infty} \rightarrow \mathbb{R}$ must coincide with $\mathbf{P}_{t}$. This follows from the fact that $\pi_{m}(U)>0$ for any nonempty set $U \in \mathcal{B}\left(\Gamma_{\infty}\right)$ which is open in the topology generated by the metric $d_{1}$. The latter can be proved as follows. For any fixed $\hat{\gamma} \in \Gamma_{\infty}$ and $\varepsilon>0$, there exist $R>0$ and $\delta>0$ such that for each $r \geq R$

$$
\begin{aligned}
& \pi_{m}\left(d_{1}(\gamma, \hat{\gamma})<\varepsilon\right) \\
& \quad \geq \pi_{m}\left(d_{\mathrm{K}}\left(\gamma_{\Lambda_{r}}, \hat{\gamma}_{\Lambda_{r}}\right)<\delta,\left|B_{1}\left(\gamma_{\Lambda_{r}}\right)-B_{1}\left(\hat{\gamma}_{\Lambda_{r}}\right)\right|<\delta, B_{1}\left(\gamma_{\Lambda_{r}^{\mathrm{c}}}\right)<\delta\right) \\
& \quad=\pi_{m}\left(d_{\mathrm{K}}\left(\gamma_{\Lambda_{r}}, \hat{\gamma}_{\Lambda_{r}}\right)<\delta,\left|B_{1}\left(\gamma_{\Lambda_{r}}\right)-B_{1}\left(\hat{\gamma}_{\Lambda_{r}}\right)\right|<\delta\right) \pi_{m}\left(B_{1}\left(\gamma_{\Lambda_{r}^{\mathrm{c}}}\right)<\delta\right) .
\end{aligned}
$$

Here, $\Lambda_{r}:=\left\{x \in X: d\left(x_{0}, x\right)<r\right\}$ and the functional $B_{1}$ is defined on the space $\Gamma_{X, 0}$ by the same formula (4.12). The first factor in (6.28) is obviously positive, while the positivity of the second factor for sufficiently big $r>0$ is implied by

$$
1=\pi_{m}\left(B_{1}(\gamma)<\infty\right)=\pi_{m}\left(\bigcup_{r=1}^{\infty}\left\{B_{1}\left(\gamma_{\Lambda_{r}^{c}}\right)<\delta\right\}\right)=\lim _{r \rightarrow \infty} \pi_{m}\left(B_{1}\left(\gamma_{\Lambda_{r}^{c}}\right)<\delta\right) .
$$




\section{$\S 7 . \quad$ Feller Property of the Heat Semigroup with Respect to the Intrinsic Metric of the Dirichlet Form}

For presenting another type of Feller property of the heat semigroup $\left(e^{-t H^{\Gamma}}\right)_{t \in \mathbb{R}_{+}}$, we shall need the space $\ddot{\Gamma}_{X}$ of all $\mathbb{Z}_{+}$-valued Radon measures $\gamma$ on $X$ such that $\gamma(X)=\infty$. This space is the closure of $\Gamma_{X}$ in the $d_{\mathrm{K}}$ metric. The space $\ddot{\Gamma}_{X}$ is equipped with the topology induced by the vague topology on $\mathcal{M}(X)$, and let $\mathcal{B}\left(\ddot{\Gamma}_{X}\right)$ denote the corresponding Borel $\sigma$-algebra.

Furthermore, let

$$
\ddot{\Gamma}_{n}:=\left\{\gamma \in \ddot{\Gamma}_{X}: B_{n}(\gamma)<\infty\right\}
$$

where $B_{n}$ is as in (4.12), but defined on all of $\ddot{\Gamma}_{X}$. Let $\ddot{\Gamma}_{\infty}:=\bigcap_{n=1}^{\infty} \ddot{\Gamma}_{n}$. We extend the metric $d_{\infty}$ to $\ddot{\Gamma}_{\infty}$ using the same formula (4.14). The Borel $\sigma$-algebra $\mathcal{B}\left(\ddot{\Gamma}_{\infty}\right)$ corresponding to the $d_{\infty}$ metric coincides with the trace $\sigma$-algebra of $\mathcal{B}\left(\ddot{\Gamma}_{X}\right)$ on $\ddot{\Gamma}_{\infty}$.

Let $\widehat{X}^{\mathbb{N}}$ denote the $\left(\mathcal{B}\left(X^{\mathbb{N}}\right)\right.$-measurable $)$ subset of $X^{\mathbb{N}}$ consisting of those $\left(x_{1}, x_{2}, \ldots\right) \in X^{\mathbb{N}}$ for which the number of the $x_{k}$ 's in any compactum in $X$ is finite. Evidently, one can identify $\ddot{\Gamma}_{X}$ with the factor space $\widehat{X}^{\mathbb{N}} / S_{\infty}$. Analogously to (4.3), we define the corresponding quotient map $I: \widehat{X}^{\mathbb{N}} \rightarrow \ddot{\Gamma}_{X}$ by

$$
\widehat{X}^{\mathbb{N}} \ni \mathbf{x}=\left(x_{1}, x_{2}, \ldots\right) \rightarrow I \mathbf{x}:=\left[x_{1}, x, \ldots\right] \in \ddot{\Gamma}_{X},
$$

which is measurable, as can be seen by similar arguments as those following (4.3).

For each $t>0$ and $\gamma \in \ddot{\Gamma}_{X}$, we define the measure $\mathbf{P}_{t, \gamma}$ on $\ddot{\Gamma}_{X}$ as the image under the mapping (7.1) of the restriction to $\widehat{X}^{\mathbb{N}}$ of any measure $\mathbb{P}_{t, \mathbf{x}}:=$ $\bigotimes_{k=1}^{\infty} p_{t, x_{k}}, \mathbf{x}=\left(x_{k}\right)_{k=1}^{\infty} \in I^{-1}\{\gamma\}$ (the resulting measure is independent of the choice of $\mathbf{x} \in I^{-1}\{\gamma\}$ ). Thus, by Lemma $4.1, \mathbf{P}_{t, \gamma}$ is either a probability measure or zero measure on $\ddot{\Gamma}_{X}$ depending on whether the series $\sum_{k=1}^{\infty} p_{t, x_{k}}(\Lambda)$ converges for each compact $\Lambda \subset X$, or not, and $\mathbf{P}_{t, \gamma}\left(\ddot{\Gamma}_{X}\right)=\mathbf{P}_{t, \gamma}\left(\Gamma_{X}\right)$. In the same way as we proved Proposition 4.2 , we conclude that, for each $\gamma \in \ddot{\Gamma}_{\infty}$,

$$
\mathbf{P}_{t, \gamma}\left(\ddot{\Gamma}_{\infty}\right)=\mathbf{P}_{t, \gamma}\left(\Gamma_{\infty}\right)=1
$$

Following [34], we introduce the $L^{2}$-Wasserstein type distance $\rho$ on $\ddot{\Gamma}_{X}$ setting, for any $\gamma_{1}=\left[x_{1}, x_{2}, \ldots\right]$ and $\gamma_{2}=\left[y_{1}, y_{2}, \ldots\right]$ from $\ddot{\Gamma}_{X}$,

$$
\rho\left(\gamma_{1}, \gamma_{2}\right):=\inf \left\{\left(\sum_{k=1}^{\infty} d\left(x_{k}, y_{\sigma(k)}\right)^{2}\right)^{1 / 2} \mid \sigma \in S_{\infty}\right\} .
$$


Notice that $\rho$ is a pseudo-metric, i.e., it takes values in $[0, \infty]$. Obviously, convergence with respect to $\rho$ implies vague convergence. We recall that $\rho$ is the intrinsic metric of the Dirichlet form obtained as the closure of $(2.8)$, see [34].

Analogously to (5.3), we set for a measurable function $F$ on $\ddot{\Gamma}_{X}$ :

$$
\left(\mathbf{P}_{t} F\right)(\gamma):=\int_{\ddot{\Gamma}_{X}} F(\xi) \mathbf{P}_{t, \gamma}(d \xi), \quad \gamma \in \ddot{\Gamma}_{X}, t>0
$$

provided the integral on the right hand side of (7.3) exists.

In the rest of this section, we shall be concerned only with the case $X=\mathbb{R}^{d}$. Let us recall that the heat kernel has now the form

$$
p(t, x, y)=(4 \pi t)^{-d / 2}\left[-\frac{1}{4 t}|x-y|^{2}\right] .
$$

We shall show that the space $C_{\rho, \mathrm{b}}\left(\ddot{\Gamma}_{\mathbb{R}^{d}}\right)$ of all bounded functions on $\ddot{\Gamma}_{\mathbb{R}^{d}}$ which are continuous with respect to the $\rho$ metric is invariant under $\mathbf{P}_{t}$ for all $t>0$.

Theorem 7.1. We have:

$$
\mathbf{P}_{t}\left(C_{\rho, \mathrm{b}}\left(\ddot{\Gamma}_{\mathbb{R}^{d}}\right)\right) \subset C_{\rho, \mathrm{b}}\left(\ddot{\Gamma}_{\mathbb{R}^{d}}\right), \quad t>0 .
$$

Proof. First, we note that the distance $\rho$ can be extended from $\ddot{\Gamma}_{\mathbb{R}^{d}}=$ $\widehat{\mathbb{R}^{d}} \mathbb{N} / S_{\infty}$ to the bigger space $\left(\mathbb{R}^{d}\right)^{\mathbb{N}} / S_{\infty}$ by using the same formula $(7.2)$ for calculating the distance between any $\gamma_{1}=\left[x_{1}, x_{2}, \ldots\right]$ and $\gamma_{2}=\left[y_{1}, y_{2}, \ldots\right]$ from $\left(\mathbb{R}^{d}\right)^{\mathbb{N}} / S_{\infty}$.

It follows directly from (7.2) that, if $\gamma_{1}$ and $\gamma_{2}$ are two elements of $\left(\mathbb{R}^{d}\right)^{\mathbb{N}} / S_{\infty}$ having finite $\rho$ distance, then $\gamma_{1} \in \ddot{\Gamma}_{\mathbb{R}^{d}}$ implies $\gamma_{2} \in \ddot{\Gamma}_{\mathbb{R}^{d}}$. Therefore, any function $F \in C_{\rho, \mathrm{b}}\left(\ddot{\Gamma}_{\mathbb{R}^{d}}\right)$ can be extended to a continuous bounded function on $\left(\mathbb{R}^{d}\right)^{\mathbb{N}} / S_{\infty}$, again denoted by $F$, as follows:

$$
F \uparrow\left(\left(\mathbb{R}^{d}\right)^{\mathbb{N}} / S_{\infty}\right) \backslash \ddot{\Gamma}_{\mathbb{R}^{d}}:=0 .
$$

Then, (7.3) yields

$$
\left(\mathbf{P}_{t} F\right)(\gamma)=\int_{\left(\mathbb{R}^{d}\right)^{\mathbb{N}}} F\left(y_{1}, y_{2}, \ldots\right) \bigotimes_{k=1}^{\infty} p_{t, x_{k}}\left(d y_{k}\right), \quad \gamma=\left[x_{1}, x_{2}, \ldots\right] \in \ddot{\Gamma}_{\mathbb{R}^{d}} .
$$

Notice that, in this formula, $F$ is considered as an $S_{\infty}$-invariant function on $\left(\mathbb{R}^{d}\right)^{\mathbb{N}}$. 
Since $F$ is bounded, so is the function $\mathbf{P}_{t} F$, and we only have to prove the continuity. To this end, let $\gamma^{j} \rightarrow \gamma$ in the $\rho$ metric. By Lemma 4.1 in [34], there always exists a representative $\left(x_{k}^{j}\right)_{k=1}^{\infty}$ of $\gamma^{j}$ such that

$$
\rho\left(\gamma^{j}, \gamma\right)=\left(\sum_{k=1}^{\infty}\left|x_{k}^{j}-x_{k}\right|^{2}\right)^{1 / 2}, \quad j \in \mathbb{N} .
$$

(7.4) and (7.5) imply

$$
\begin{gathered}
\left(\mathbf{P}_{t} F\right)\left(\gamma^{j}\right)=\int_{\left(\mathbb{R}^{d}\right)^{\mathbb{N}}} F\left(y_{1}+x_{1}^{j}, y_{2}+x_{2}^{j}, \ldots\right) \bigotimes_{k=1}^{\infty} p_{t}\left(d y_{k}\right), \\
p_{t}(d y)=(4 \pi t)^{-d / 2} \exp \left[-\frac{1}{4 t}|y|^{2}\right] d y
\end{gathered}
$$

Since the integrand in (7.7) is a bounded function, it suffices to show that for any fixed $\left(y_{k}\right)_{k=1}^{\infty} \in\left(\mathbb{R}^{d}\right)^{\mathbb{N}}$

$$
F\left(y_{1}+x_{1}^{j}, y_{2}+x_{2}^{j}, \ldots\right) \rightarrow F\left(y_{1}+x_{1}, y_{2}+x_{2}, \ldots\right) \text { as } j \rightarrow \infty .
$$

But this follows from the fact that $F$ is continuous in the $\rho$ metric and from the convergence

$$
\begin{aligned}
& \rho\left(\left[y_{1}+x_{1}^{j}, y_{2}+x_{2}^{j}, \ldots\right],\left[y_{1}+x_{1}, y_{2}+x_{2}, \ldots\right]\right) \\
& \quad \leq\left(\sum_{k=1}^{\infty}\left|y_{k}+x_{k}^{j}-y_{k}-x_{k}\right|^{2}\right)^{1 / 2}=\rho\left(\gamma_{j}, \gamma\right) \rightarrow 0
\end{aligned}
$$

as $j \rightarrow \infty$, which, in turn, is implied by (7.6).

Remark 7.1. Theorem 7.1, in particular, yields that if $\ddot{\Gamma}_{\mathbb{R}^{d}}$ is of full $\mathbf{P}_{t, \gamma}$ measure for some $\gamma \in \ddot{\Gamma}_{\mathbb{R}^{d}}$, then it is also of full $\mathbf{P}_{t, \gamma^{\prime}}$ measure for each $\gamma^{\prime} \in$ $\ddot{\Gamma}_{\mathbb{R}^{d}}$ such that $\rho\left(\gamma, \gamma^{\prime}\right)<\infty$. For it suffices to note that $1 \in C_{\rho, \mathrm{b}}\left(\ddot{\Gamma}_{\mathbb{R}^{d}}\right)$ and $\left(\mathbf{P}_{t} 1\right)(\gamma)=\mathbf{P}_{t, \gamma}\left(\ddot{\Gamma}_{X}\right)$.

Finally, we shall present another version of the latter theorem. Let $C_{\rho, \mathrm{b}}\left(\ddot{\Gamma}_{\infty}\right)$ denote the space of all bounded functions on $\ddot{\Gamma}_{\infty}$ that are continuous with respect to the $\rho$ metric. It is easy to see that each element of $\left(\mathbb{R}^{d}\right)^{\mathbb{N}} / S_{\infty}$ having a finite distance to $\ddot{\Gamma}_{\infty}$ itself belongs to $\ddot{\Gamma}_{\infty}$. Therefore, any function $F \in C_{\rho, \mathrm{b}}\left(\ddot{\Gamma}_{\infty}\right)$ can be extended to a function from $C_{\rho, \mathrm{b}}\left(\ddot{\Gamma}_{\mathbb{R}^{d}}\right)$ by setting $F$ to be equal to zero on $\ddot{\Gamma}_{\mathbb{R}^{d}} \backslash \ddot{\Gamma}_{\infty}$. We note that the convergence on $\ddot{\Gamma}_{\infty}$ with respect to the $\rho$ metric implies the convergence with respect to the $d_{\infty}$ metric.

Since for each $\gamma \in \ddot{\Gamma}_{\infty}$ the measure $\mathbf{P}_{t, \gamma}$ is concentrated on $\ddot{\Gamma}_{\infty}$, we get the following corollary of Theorem 7.1: 
Corollary 7.1. We have:

$$
\mathbf{P}_{t}\left(C_{\rho, \mathrm{b}}\left(\ddot{\Gamma}_{\infty}\right)\right) \subset C_{\rho, \mathrm{b}}\left(\ddot{\Gamma}_{\infty}\right), \quad t>0
$$

\section{$\S 8$. Brownian Motion on the Configuration Space}

We again consider the case of a general manifold $X$. Analogously to (5.4), (5.5), we define the family of kernels $\left(\mathbf{P}_{t}\right)_{t \in \mathbb{R}_{+}}$on the space $\left(\ddot{\Gamma}_{\infty}, \mathcal{B}\left(\ddot{\Gamma}_{\infty}\right)\right)$ setting

$$
\mathbf{P}_{t}(\gamma, A):=\mathbf{P}_{t, \gamma}(A), \quad \gamma \in \ddot{\Gamma}_{\infty}, A \in \mathcal{B}\left(\ddot{\Gamma}_{\infty}\right), t \in \mathbb{R}_{+},
$$

where $\mathbf{P}_{t, \gamma}, t>0, \gamma \in \ddot{\Gamma}_{X}$, is defined as in the previous section and

$$
\mathbf{P}_{0, \gamma}:=\varepsilon_{\gamma} .
$$

Analogously to Proposition 5.1, we conclude that $\left(\mathbf{P}_{t}\right)_{t \in \mathbb{R}_{+}}$is a Markov semigroup of kernels on $\left(\ddot{\Gamma}_{\infty}, \mathcal{B}\left(\ddot{\Gamma}_{\infty}\right)\right)$.

In this section, we shall give a direct construction of the independent infinite particle process. Under some additional conditions on the manifold $X$, we shall show that the resulting process is the unique continuous Markov process on $\ddot{\Gamma}_{\infty}$ with transition probabilities $\mathbf{P}_{t}(\gamma, \cdot)$. (We note that we are forced to deal with the space $\ddot{\Gamma}_{\infty}$, rather than $\Gamma_{\infty}$, because in the general case we cannot exclude collision of the particles, see Corollary 8.1 below).

First, we strengthen a little bit condition (C1) by requiring the following stronger upper bound:

$\left(\mathrm{C} 1^{\prime}\right)$ For each $t>0$, there exist $\vartheta_{t} \in(0, t), C_{t}>0$, and $\varepsilon_{t}>0$ such that

$$
p(s, x, y) \leq C_{t} \exp \left[-d(x, y)^{1+\varepsilon_{t}}\right], \quad s \in\left(t-\vartheta_{t}, t+\vartheta_{t}\right), x, y \in X .
$$

Evidently, (4.9) and (4.11) imply $\left(\mathrm{C1}^{\prime}\right)$.

Let us introduce the function

$$
\tau(\delta, r):=\sup _{t \in(0, \delta]} \sup _{x \in X} \int_{B(x, r)^{\mathrm{c}}} p(t, x, y) m(d y), \quad \delta>0, r>0 .
$$

Because of $(2.2), \tau(\delta, r) \leq 1$ for all $\delta, r>0$, and for each fixed $r>0 \tau(\cdot, r)$ is an increasing function on $(0, \infty)$.

Let $\Omega:=C\left(\mathbb{R}_{+} ; X\right)$ denote the space of all continuous functions (paths) from $\mathbb{R}_{+}$to $X$, and let $\mathcal{F}$ be the product $\sigma$-algebra on $\Omega$, i.e.,

$$
\mathcal{F}:=\sigma\left\{x_{t}, t \in \mathbb{R}_{+}\right\},
$$

where $\Omega \ni \omega \mapsto x_{t}(\omega):=\omega(t) \in X$. For each $x \in X$, let $P_{x}$ denote the measure on $(\Omega, \mathcal{F})$ corresponding to Brownian motion on $X$ starting at $x$.

We shall need the following lemma. 
Lemma 8.1. Let $0 \leq a<b$ with $b-a \leq \delta$. Then, for each $x \in X$ and $r>0$,

$$
\left.P_{x}(\exists s, t \in[a, b]: d(\omega(s), \omega(t))>r)\right) \leq 2 \tau\left(\delta, \frac{1}{4} r\right)
$$

Proof. This lemma is a straightforward generalization of [30, Appendix A, Lemma 4], which deals with the usual Brownian motion on $\mathbb{R}^{d}$. However, for completeness, we present a proof of this lemma in the Appendix.

We suppose:

(C3) For each fixed $r>0$,

$$
\tau(\delta, r) \rightarrow 0 \quad \text { as } \delta \rightarrow 0,
$$

and there exist $\widetilde{\delta}>0$ and $C>0$ such that

$$
\tau(\widetilde{\delta}, r) \leq C e^{-r}, \quad r>0
$$

The following simple lemma gives a sufficient condition for (C3) to hold.

Lemma 8.2. Suppose that the manifold $X$ has nonnegative Ricci curvature and the heat kernel $p(t, x, y)$ of $X$ satisfies the Gaussian upper bound for small values of $t$ :

$$
p(t, x, y) \leq C t^{-n / 2} \exp \left[-\frac{d(x, y)^{2}}{D t}\right], \quad t \in(0, \widetilde{\delta}], x, y \in X
$$

where $n \in \mathbb{N}$ and $\widetilde{\delta}, C$, and $D$ are positive constants. Then, (C3) is satisfied.

Remark 8.1. Concerning the Gaussian upper bound (8.4), see e.g. [10], [14], [15] and the references therein. In particular, (8.4) is implied by the estimate

$$
p(x, x, t) \leq C t^{-n / 2}, \quad t>0, x \in X .
$$

Proof of Lemma 8.2. Fix $r>0$, then for $t \in(0, \widetilde{\delta}]$ and $x \in X$ we get, by (4.10) and (8.4),

$$
\int_{B(x, r)^{\mathrm{c}}} p(t, x, y) m(d y)
$$




$$
\begin{aligned}
& \leq \int_{B(x, r)^{\mathrm{c}}} C t^{-n / 2} \exp \left[-\frac{d(x, y)^{2}}{D t}\right] m(d y) \\
& \leq \text { const }_{1} \int_{B(x, r)^{\mathrm{c}}} \exp \left[\frac{1}{t}\left(\frac{r^{2}}{2 D}-\frac{d(x, y)^{2}}{D}\right)\right] m(d y) \\
& \leq \text { const }_{1} \sum_{n=1}^{\infty} \exp \left[\frac{1}{t}\left(\frac{r^{2}}{2 D}-\frac{(r+n-1)^{2}}{D}\right)\right] m(B(x, r+n) \backslash B(x, r+n-1)) \\
& \leq \text { const }_{2} \sum_{n=1}^{\infty} \exp \left[\frac{1}{t}\left(\frac{r^{2}}{2 D}-\frac{(r+n-1)^{2}}{D}\right)\right](r+n)^{d} .
\end{aligned}
$$

Since each term of the latter series monotonically converges to zero as $t \rightarrow 0$, we get (8.2).

Next, because $\tau(\delta, r)$ is bounded by 1 , it is enough to verify that (8.3) holds for all $r \geq R$ with some $R>0$. Now, analogously to (8.5), we get for each $t \in(0, \widetilde{\delta}]$ and $r \geq 1$

$$
\begin{gathered}
\int_{B(x, r)^{\mathrm{c}}} p(t, x, y) m(d y) \leq \mathrm{const}_{3} \sum_{n=1}^{\infty} \exp \left[\frac{1}{\widetilde{\delta}}\left(\frac{1}{2 D}-\frac{(r+n-1)^{2}}{D}\right)\right](r+n)^{d} \\
\leq \operatorname{const}_{4} \sum_{n=1}^{\infty} \exp [-2(r+n-1)+(r+n)]=\text { const }_{4} e^{-r} \sum_{n=1}^{\infty} e^{-n}
\end{gathered}
$$

which yields the statement.

Theorem 8.1. Let $\left(\mathrm{C}^{\prime}\right),(\mathrm{C} 2)$ and $(\mathrm{C} 3)$ hold. Then, the independent infinite particle process can be realized as the unique continuous, time homogeneous Markov process

$$
\mathbf{M}=\left(\boldsymbol{\Omega}, \mathbf{F},\left(\mathbf{F}_{t}\right)_{t \in \mathbb{R}_{+}},\left(\boldsymbol{\theta}_{t}\right)_{t \in \mathbb{R}_{+}},\left(\mathbf{P}_{\gamma}\right)_{\gamma \in \ddot{\Gamma}_{\infty}},\left(\mathbf{X}_{t}\right)_{t \in \mathbb{R}_{+}}\right)
$$

on the state-space $\left(\ddot{\Gamma}_{\infty}, \mathcal{B}\left(\ddot{\Gamma}_{\infty}\right)\right)$ with transition probability function $\left(\mathbf{P}_{t}\right)_{t \in \mathbb{R}_{+}}$ (cf. e.g. $[9])$.

Proof of Theorem 8.1. Let us consider the set $\Omega^{\mathbb{N}}$ and the product $\sigma$ algebra $\mathcal{C}_{\sigma}\left(\Omega^{\mathbb{N}}\right)$ on it that is constructed from the $\sigma$-algebra $\mathcal{F}$ on $\Omega$.

We fix any $\mathbf{x}=\left(x_{k}\right)_{k=1}^{\infty} \in \widehat{X}^{\mathbb{N}}$ such that

$$
\sum_{k=1}^{\infty} \exp \left[-\frac{1}{n} d\left(x_{0}, x_{k}\right)\right]<\infty \quad \forall n \in \mathbb{N}
$$

and define the product measure

$$
\mathbb{P}_{\mathbf{x}}:=\bigotimes_{k=1}^{\infty} P_{x_{k}}
$$


on $\left(\Omega^{\mathbb{N}}, \mathcal{C}_{\sigma}\left(\Omega^{\mathbb{N}}\right)\right)$.

By using (4.16), we conclude from (8.6) that

$$
\begin{aligned}
\int_{X^{\mathbb{N}}} & \sum_{k=1}^{\infty} \exp \left[-\frac{1}{n} d\left(x_{0}, y_{k}\right)\right] \bigotimes_{k=1}^{\infty} p_{t, x_{k}}\left(d y_{k}\right) \\
& =\sum_{k=1}^{\infty} \int_{X} \exp \left[-\frac{1}{n} d\left(x_{0}, y\right)\right] p_{t, x_{k}}(d y)<\infty
\end{aligned}
$$

for all $t>0$ and $n \in \mathbb{N}$, which yields that, for each fixed $t \in \mathbb{R}_{+}$,

$$
\begin{aligned}
& \sum_{k=1}^{\infty} \exp \left[-\frac{1}{n} d\left(x_{0}, \omega_{k}(t)\right)\right]<\infty \\
& \quad \text { for all } n \in \mathbb{N} \text { and } \mathbb{P}_{\mathbf{x}} \text {-a.e. } \omega=\left(\omega_{k}\right)_{k=1}^{\infty} \in \Omega^{\mathbb{N}}, \\
& \quad \text { in particular, }\left(\omega_{k}(t)\right)_{k=1}^{\infty} \in \widehat{X}^{\mathbb{N}} \text { for such } \omega \in \Omega^{\mathbb{N}}
\end{aligned}
$$

Lemma 8.3. For each fixed $t \in \mathbb{R}_{+}$and $\mathbf{x}=\left(x_{k}\right)_{k=1}^{\infty} \in \widehat{X}^{\mathbb{N}}$ satisfying (8.6), we have

$$
\mathbb{P}_{\mathbf{x}}\left(\bigcup_{i=1}^{\infty} \bigcap_{k=1}^{\infty}\left\{d\left(\omega_{k}(s), \omega_{k}(t)\right) \leq \max \left\{1, \frac{1}{2} d\left(x_{0}, \omega_{k}(t)\right)\right\} \forall s \in\left(t, t+\frac{1}{i}\right]\right\}\right)=1 \text {. }
$$

Proof. First, we will prove (8.9) for $t=0$. Thus, we have to show that

$$
\mathbb{P}_{\mathbf{x}}\left(\bigcup_{i=1}^{\infty} \bigcap_{k=1}^{\infty}\left\{d\left(\omega_{k}(s), x_{k}\right) \leq \max \left\{1, \frac{1}{2} d\left(x_{0}, x_{k}\right)\right\} \forall s \in\left(0, \frac{1}{i}\right]\right\}\right)=1,
$$

or equivalently

$$
\mathbb{P}_{\mathbf{x}}\left(\bigcap_{i=1}^{\infty} \bigcup_{k=1}^{\infty}\left\{\exists s \in\left(0, \frac{1}{i}\right]: d\left(\omega_{k}(s), x_{k}\right)>\max \left\{1, \frac{1}{2} d\left(x_{0}, x_{k}\right)\right\}\right\}\right)=0 .
$$

Since

$$
\mathbb{P}_{\mathbf{x}}\left(\bigcap_{i=1}^{\infty} \bigcup_{k=1}^{\infty}\left\{\exists s \in\left(0, \frac{1}{i}\right]: d\left(\omega_{k}(s), x_{k}\right)>\max \left\{1, \frac{1}{2} d\left(x_{0}, x_{k}\right)\right\}\right\}\right)
$$




$$
=\lim _{i \rightarrow \infty} \mathbb{P}_{\mathbf{x}}\left(\bigcup_{k=1}^{\infty}\left\{\exists s \in\left(0, \frac{1}{i}\right]: d\left(\omega_{k}(s), x_{k}\right)>\max \left\{1, \frac{1}{2} d\left(x_{0}, x_{k}\right)\right\}\right\}\right)
$$

we have, by Lemma 8.1,

$$
\begin{aligned}
\mathbb{P}_{\mathbf{x}}( & \left.\bigcup_{k=1}^{\infty}\left\{\exists s \in\left(0, \frac{1}{i}\right]: d\left(\omega_{k}(s), x_{k}\right)>\max \left\{1, \frac{1}{2} d\left(x_{0}, x_{k}\right)\right\}\right\}\right) \\
& \leq \sum_{k=1}^{\infty} \mathbb{P}_{\mathbf{x}}\left(\exists s \in\left(0, \frac{1}{i}\right]: d\left(\omega_{k}(s), x_{k}\right)>\max \left\{1, \frac{1}{2} d\left(x_{0}, x_{k}\right)\right\}\right) \\
& =\sum_{k=1}^{\infty} P_{x_{k}}\left(\exists s \in\left(0, \frac{1}{i}\right]: d\left(\omega(s), x_{k}\right)>\max \left\{1, \frac{1}{2} d\left(x_{0}, x_{k}\right)\right\}\right) \\
& \leq 2 \sum_{k=1}^{\infty} \tau\left(\frac{1}{i}, \max \left\{\frac{1}{4}, \frac{1}{8} d\left(x_{0}, x_{k}\right)\right\}\right) .
\end{aligned}
$$

By (8.2),

$$
\tau\left(\frac{1}{i}, \max \left\{\frac{1}{4}, \frac{1}{8} d\left(x_{0}, x_{k}\right)\right\}\right) \rightarrow 0 \quad \text { as } i \rightarrow \infty
$$

for each $x_{k}$. On the other hand, it follows from (8.3) that, for any $i \in \mathbb{N}$ satisfying $(1 / i) \leq \widetilde{\delta}$, the latter series in (8.13) is majorized by the series

$$
2 C \sum_{k=1}^{\infty} \exp \left[-\max \left\{\frac{1}{4}, \frac{1}{8} d\left(x_{0}, x_{k}\right)\right\}\right]
$$

which converges due to (8.6). Hence, (8.11) follows from (8.12)-(8.14), and the monotone convergence theorem.

Next, using the Markov property of Brownian motion on $X$, we easily conclude that

$$
\begin{aligned}
& \mathbb{P}_{\mathbf{x}}\left(\bigcup_{i=1}^{\infty} \bigcap_{k=1}^{\infty}\left\{d\left(\omega_{k}(s), \omega_{k}(t)\right) \leq \max \left\{1, \frac{1}{2} d\left(x_{0}, \omega_{k}(t)\right)\right\} \forall s \in\left(t, t+\frac{1}{i}\right]\right\}\right) \\
& =\int_{\widehat{X}^{\mathbb{N}}} \mathbb{P}_{t, \mathbf{x}}(d \mathbf{y}) \mathbb{P}_{\mathbf{y}}\left(\bigcup_{i=1}^{\infty} \bigcap_{k=1}^{\infty}\left\{d\left(\omega_{k}(s), y_{k}\right) \leq \max \left\{1, \frac{1}{2} d\left(x_{0}, y_{k}\right)\right\} \forall s \in\left(0, \frac{1}{i}\right]\right\}\right),
\end{aligned}
$$

where $\mathbb{P}_{t, \mathbf{x}}$ is the distribution of $\omega(t)=\left(\omega_{k}(t)\right)_{k=1}^{\infty}$ under $\mathbb{P}_{\mathbf{x}}$. Now, (8.9) follows from (8.8), (8.10) and (8.15). 
Lemma 8.4. For each fixed $t>0$ and $\mathbf{x}=\left(x_{k}\right)_{k=1}^{\infty} \in \widehat{X}^{\mathbb{N}}$ satisfying (8.6), we have

$$
\begin{aligned}
& \mathbb{P}_{\mathbf{x}}\left(\bigcup _ { i = \mathcal { I } _ { t } } ^ { \infty } \bigcap _ { k = 1 } ^ { \infty } \left\{d\left(\omega_{k}\left(t-\frac{1}{i}\right), \omega_{k}(s)\right)\right.\right. \\
& \left.\left.\quad \leq \max \left\{1, \frac{1}{2} d\left(x_{0}, \omega_{k}\left(t-\frac{1}{i}\right)\right)\right\} \forall s \in\left(t-\frac{1}{i}, t\right]\right\}\right)=1,
\end{aligned}
$$

where $\mathcal{I}_{t}:=\left[t^{-1}\right]+1([a]$ denoting the integer part of $a>0)$.

Proof. It is enough to show that

$$
\begin{aligned}
& \lim _{i \rightarrow \infty} \mathbb{P}_{\mathbf{x}}\left(\bigcup _ { k = 1 } ^ { \infty } \left\{\exists s \in\left(t-\frac{1}{i}, t\right]: d\left(\omega_{k}\left(t-\frac{1}{i}\right), \omega_{k}(s)\right)\right.\right. \\
&\left.\left.>\max \left\{1, \frac{1}{2} d\left(x_{0}, \omega_{k}\left(t-\frac{1}{i}\right)\right)\right\}\right\}\right)=0 .
\end{aligned}
$$

Using Lemma 8.1, we get

$$
\begin{aligned}
\mathbb{P}_{\mathbf{x}}\left(\bigcup _ { k = 1 } ^ { \infty } \left\{\exists s \in\left(t-\frac{1}{i}, t\right]: d\left(\omega_{k}\left(t-\frac{1}{i}\right), \omega_{k}(s)\right)\right.\right. \\
\left.\left.\quad>\max \left\{1, \frac{1}{2} d\left(x_{0}, \omega_{k}\left(t-\frac{1}{i}\right)\right)\right\}\right\}\right) \\
\leq \sum_{k=1}^{\infty} P_{x_{k}}\left(\exists s \in\left(t-\frac{1}{i}, t\right]: d\left(\omega\left(t-\frac{1}{i}\right), \omega(s)\right)\right. \\
\left.\quad>\max \left\{1, \frac{1}{2} d\left(x_{0}, \omega\left(t-\frac{1}{i}\right)\right)\right\}\right) \\
=\sum_{k=1}^{\infty} \int_{X} m(d y) p\left(t-\frac{1}{i}, x_{k}, y\right) P_{y}\left(\exists s \in\left(0, \frac{1}{i}\right]: d(y, \omega(s))\right. \\
\left.\quad>\max \left\{1, \frac{1}{2} d\left(x_{0}, y\right)\right\}\right) \\
\leq \sum_{k=1}^{\infty} \int_{X} m(d y) p\left(t-\frac{1}{i}, x_{k}, y\right) 2 \tau\left(\frac{1}{i}, \max \left\{\frac{1}{4}, \frac{1}{8} d\left(x_{0}, y\right)\right\}\right) .
\end{aligned}
$$

Now, it follows from (8.2) that

$$
p\left(t-\frac{1}{i}, x_{k}, y\right) 2 \tau\left(\frac{1}{i}, \max \left\{\frac{1}{4}, \frac{1}{8} d\left(x_{0}, y\right)\right\}\right) \rightarrow 0 \quad \text { as } i \rightarrow \infty
$$


for each $x_{k}$ and $y \in X$. Next, by $\left(\mathrm{C}^{\prime}\right)$ and (8.3), we have, for $i>\max \left\{\vartheta_{t}^{-1}\right.$, $\left.\widetilde{\delta}^{-1}\right\}$,

$$
\begin{aligned}
p(t & \left.-\frac{1}{i}, x_{k}, y\right) 2 \tau\left(\frac{1}{i}, \max \left\{\frac{1}{4}, \frac{1}{8} d\left(x_{0}, y\right)\right\}\right) \\
& \leq C_{t} \exp \left[-d\left(x_{k}, y\right)^{1+\varepsilon_{t}}\right] 2 C \exp \left[-\max \left\{\frac{1}{4}, \frac{1}{8} d\left(x_{0}, y\right)\right\}\right] \\
& \leq \text { const } \exp \left[-\frac{1}{16} d\left(x_{k}, y\right)-\max \left\{\frac{1}{4}, \frac{1}{8} d\left(x_{0}, y\right)\right\}\right] \\
& \leq \text { const } \exp \left[-\frac{1}{16} d\left(x_{0}, x_{k}\right)\right] \exp \left[\frac{1}{16} d\left(x_{0}, y\right)-\max \left\{\frac{1}{4}, \frac{1}{8} d\left(x_{0}, y\right)\right\}\right] .
\end{aligned}
$$

Hence, by (8.6), (8.17)-(8.19), (4.15), and the dominated convergence theorem, we get (8.16).

From (8.6), (8.8), and Lemmas 8.3 and 8.4, we get the central lemma of the proof:

Lemma 8.5. Let $x \in \widehat{X}^{\mathbb{N}}$ satisfy (8.6). For $t>0$ and $i \geq \mathcal{I}_{t}$, we set

$$
\begin{aligned}
& \mathbb{A}_{t, i}:=\left[\bigcup _ { l = \mathcal { I } _ { t } } ^ { i } \left\{\omega \in \Omega^{\mathbb{N}}: \sum_{k=1}^{\infty} \exp \left[-\frac{1}{n} d\left(x_{0}, \omega_{k}\left(t-\frac{1}{l}\right)\right)\right]<\infty \forall n \in \mathbb{N},\right.\right. \\
& d\left(\omega_{k}\left(t-\frac{1}{l}\right), \omega_{k}(s)\right) \\
& \left.\left.\quad \leq \max \left\{1, \frac{1}{2} d\left(x_{0}, \omega_{k}\left(t-\frac{1}{l}\right)\right)\right\} \forall s \in\left(t-\frac{1}{l}, t\right] \forall k \in \mathbb{N}\right\}\right] \\
& \bigcap\left\{\omega \in \Omega^{\mathbb{N}}: \sum_{k=1}^{\infty} \exp \left[-\frac{1}{n} d\left(x_{0}, \omega_{k}(t)\right)\right]<\infty \forall n \in \mathbb{N},\right. \\
& \left.d\left(\omega_{k}(t), \omega_{k}(s)\right) \leq \max \left\{1, \frac{1}{2} d\left(x_{0}, \omega_{k}(t)\right) \forall s \in\left(t, t+\frac{1}{i}\right], \forall k \in \mathbb{N}\right\}\right\},
\end{aligned}
$$

and for $t=0$ and $i \in \mathbb{N}$, we set

$$
\begin{aligned}
\mathbb{A}_{t, i} & =\mathbb{A}_{0, i} \\
& :=\left\{\omega \in \Omega^{\mathbb{N}}: d\left(x_{k}, \omega_{k}(s)\right) \leq \max \left\{1, \frac{1}{2} d\left(x_{0}, x_{k}\right)\right\} \forall s \in\left(0, \frac{1}{i}\right] \forall k \in \mathbb{N}\right\} .
\end{aligned}
$$

Then,

$$
\mathbb{P}_{\mathbf{x}}\left(\bigcup_{i=\mathcal{I}_{t}}^{\infty} \mathbb{A}_{t, i}\right)=\lim _{i \rightarrow \infty} \mathbb{P}_{\mathbf{x}}\left(\mathbb{A}_{t, i}\right)=1
$$

for each $t \in \mathbb{R}_{+}$. 
Let $D:=\mathbb{R}_{+} \cap \mathbb{Q}(\mathbb{Q}$ denoting the set of rational numbers), and $D=$ $\left\{t_{l}\right\}_{l=1}^{\infty}$. We consider arbitrary numbers $\varepsilon_{l p}>0, l, p \in \mathbb{N}$, such that $\sum_{l=1}^{\infty} \varepsilon_{l p}<$ $\infty$ for each $p \in \mathbb{N}$, and

$$
\lim _{p \rightarrow \infty} \sum_{l=1}^{\infty} \varepsilon_{l p}=0 .
$$

For each $l, p \in \mathbb{N}$, we choose an $i_{l p} \in \mathbb{N}$ such that

$$
\mathbb{P}_{\mathbf{x}}\left(\mathbb{A}_{t_{l}, i_{l p}}^{\mathrm{c}}\right) \leq \varepsilon_{l p}
$$

which exists due to Lemma 8.5. Then, we set

$$
\mathbb{A}_{p}:=\bigcap_{l=1}^{\infty} \mathbb{A}_{t_{l}, i_{l p}} .
$$

By (8.20), (8.21) and (8.22),

$$
\lim _{p \rightarrow \infty} \mathbb{P}_{\mathbf{x}}\left(\mathbb{A}_{p}\right)=1
$$

For each $p \in \mathbb{N}$, let us consider the set

$$
T_{p}:=\mathbb{R}_{+} \cap\left[\bigcup_{l=1}^{\infty}\left(t_{l}-i_{l p}^{-1}, t_{l}+i_{l p}^{-1}\right)\right] .
$$

Since the set $\bigcup_{l=1}^{\infty}\left(t_{l}-i_{l p}^{-1}, t_{l}+i_{l p}^{-1}\right)$ is open in $\mathbb{R}$, and since $T_{p}$ is dense in $\mathbb{R}_{+}$, we have $T_{p}=\mathbb{R}_{+} \backslash T_{p}^{\mathrm{c}}$, where $T_{p}^{\mathrm{c}}$ is countable. We set

$$
\mathbf{A}_{p}:=\mathbb{A}_{p} \cap\left[\bigcap_{t \in T_{p}^{\mathrm{c}}} \bigcup_{i=\mathcal{I}_{t}}^{\infty} \mathbb{A}_{t, i}\right] .
$$

By Lemma 8.5, we get

$$
\mathbb{P}_{\mathbf{x}}\left(\mathbf{A}_{p}\right)=\mathbb{P}_{\mathbf{x}}\left(\mathbb{A}_{p}\right)
$$

Finally, we set

$$
\mathbf{A}:=\bigcup_{p=1}^{\infty} \mathbf{A}_{p} .
$$

Therefore, by (8.23) and (8.24), we get

$$
\mathbb{P}_{\mathbf{x}}(\mathbf{A})=1 .
$$


Lemma 8.6. For any $\omega \in \mathbf{A}$ and $n \in \mathbb{N}$, we have

$$
\forall t \in \mathbb{R}_{+}: \quad B_{n}(\omega(t)):=\sum_{k=1}^{\infty} \exp \left[-\frac{1}{n} d\left(x_{0}, \omega_{k}(t)\right)\right]<\infty
$$

and moreover, the mapping

$$
\mathbb{R} \ni t \mapsto B_{n}(\omega(t)) \in \mathbb{R}
$$

is continuous.

Proof. We note that, if $B_{2 n}(\omega(t))<\infty$ and if there exists an interval $(a, b) \subset \mathbb{R}_{+}$such that $t \in[a, b]$ and

$$
d\left(\omega_{k}(s), \omega_{k}(t)\right) \leq \max \left\{1, \frac{1}{2} d\left(x_{0}, \omega_{k}(t)\right)\right\}, \quad s \in(a, b),
$$

then the series

$$
\sum_{k=1}^{\infty} \exp \left[-\frac{1}{n} d\left(x_{0}, \omega_{k}(s)\right)\right], \quad s \in(a, b),
$$

are majorized by the convergent series

$$
\sum_{k=1}^{\infty} \exp \left[-\frac{1}{2 n} d\left(x_{0}, \omega_{k}(t)\right)\right] \text {. }
$$

Hence, the statement follows from the construction of the set $\mathbf{A}$.

Now, we define the action of the group $S_{\infty}$ on $\Omega^{\mathbb{N}}$ by

$$
\sigma\left(\left(\omega_{k}\right)_{k=1}^{\infty}\right):=\left(\omega_{\sigma(k)}\right)_{k=1}^{\infty}, \quad \sigma \in S_{\infty} .
$$

Evidently, the set $\mathbf{A}$ is invariant under the action of $S_{\infty}$. We introduce the factor space $\Omega^{\mathbb{N}} / S_{\infty}$ consisting of factor classes $\left[\omega_{1}, \omega_{2}, \ldots\right]$. Analogously to (4.3) and (7.1), we introduce then the mapping

$$
\Omega^{\mathbb{N}} \ni \omega=\left(\omega_{k}\right)_{k=1}^{\infty} \mapsto \mathbf{I} \omega=\left[\omega_{1}, \omega_{2}, \ldots\right] \in \Omega^{\mathbb{N}} / S_{\infty} .
$$

Lemma 8.7. We have

$$
\mathbf{I A} \subset C\left(\mathbb{R}_{+} ; \ddot{\Gamma}_{\infty}\right)
$$

where $C\left(\mathbb{R}_{+} ; \ddot{\Gamma}_{\infty}\right)$ denotes the set of continuous mappings from $\mathbb{R}_{+}$into $\ddot{\Gamma}_{\infty}$. 
Proof. Taking notice of Lemma 8.6 and of the definition of the metric space $\ddot{\Gamma}_{\infty}$, it remains only to show that, for each fixed $\left(\omega_{k}\right)_{k=1}^{\infty} \in \mathbf{A}$, the mapping

$$
\mathbb{R}_{+} \ni t \mapsto\left\{\omega_{k}(t)\right\}_{k=1}^{\infty} \in \ddot{\Gamma}_{\infty}
$$

is vaguely continuous.

To this end, let us fix any $t \in \mathbb{R}_{+}$and any ball $B\left(x_{0}, r\right)$ of radius $r>0$. Then, there exist $\varepsilon>0$ and $K \in \mathbb{N}$ such that

$$
\sum_{k=K}^{\infty} \exp \left[-d\left(x_{0}, \omega_{k}(s)\right)\right]<e^{-r}, \quad s \in \mathbb{R}_{+} \cap(t-\varepsilon, t+\varepsilon)
$$

(see the proof of Lemma 8.6). Hence,

$$
\omega_{k}(s) \notin B\left(x_{0}, r\right), \quad k \geq K, s \in \mathbb{R}_{+} \cap(t-\varepsilon, t+\varepsilon),
$$

which, together with the continuity of each $\omega_{k}$ as a mapping from $\mathbb{R}_{+}$into $X$, implies the vague continuity of (8.27).

Thus, by Lemma 8.7, we have that

$$
\mathbf{I}: \mathbf{A} \rightarrow \Omega:=C\left(\mathbb{R}_{+} ; \ddot{\Gamma}_{\infty}\right) .
$$

Denote the trace $\sigma$-algebra of $\mathcal{C}_{\sigma}\left(\Omega^{\mathbb{N}}\right)$ on $\mathbf{A}$ by $\mathcal{C}_{\sigma}(\mathbf{A})$. Let $\mathbf{F}$ be the product $\sigma$-algebra on $\boldsymbol{\Omega}=C\left(\mathbb{R}_{+} ; \ddot{\Gamma}_{\infty}\right)$ generated by the $\sigma$-algebra $\mathcal{B}\left(\ddot{\Gamma}_{\infty}\right)$ :

$$
\mathbf{F}:=\sigma\left\{\mathbf{X}_{t}, t \in \mathbb{R}_{+}\right\}
$$

where

$$
\mathbf{X}_{t}(\omega):=\omega(t)
$$

Since the mapping $I: \widehat{X}^{\mathbb{N}} \rightarrow \ddot{\Gamma}_{X}$ defined by (7.1) is measurable and since $\mathcal{B}\left(\ddot{\Gamma}_{\infty}\right)$ is the trace $\sigma$-algebra of $\mathcal{B}\left(\ddot{\Gamma}_{X}\right)$ on $\ddot{\Gamma}_{\infty}$, we easily conclude that the mapping $\mathbf{I}$ is $\mathcal{C}_{\sigma}(\mathbf{A})$-F-measurable. Because of (8.26), we can consider $\mathbb{P}_{\mathbf{x}}$ as a probability measure on $\left(\mathbf{A}, \mathcal{C}_{\sigma}(\mathbf{A})\right)$ and let $\mathbf{P}_{\mathbf{x}}$ denote the image of this measure under the mapping $\mathbf{I}$. Thus, $\mathbf{P}_{\mathbf{x}}$ is a probability measure on $(\boldsymbol{\Omega}, \mathbf{F})$.

For each $\sigma \in S_{\infty}$, the measures $\mathbf{P}_{\mathbf{x}}$ and $\mathbf{P}_{\sigma(\mathbf{x})}$ evidently coincide, and so for each $\gamma \in \ddot{\Gamma}_{\infty}$, we can introduce the probability measure $\mathbf{P}_{\gamma}:=\mathbf{P}_{\mathbf{x}}$, where $\mathbf{x}$ is an arbitrary element of the set $I^{-1} \gamma$.

Finally, we introduce the sub- $\sigma$-algebras $\mathbf{F}_{t}:=\sigma\left\{\mathbf{X}_{s}, s \leq t\right\}$ and the translations $\left(\boldsymbol{\theta}_{t} \omega\right)(s):=\omega(s+t), t \in \mathbb{R}_{+}$. Thus, we get

$$
\left(\boldsymbol{\Omega}, \mathbf{F},\left(\mathbf{F}_{t}\right)_{t \in \mathbb{R}_{+}},\left(\boldsymbol{\theta}_{t}\right)_{t \in \mathbb{R}_{+}},\left(\mathbf{P}_{\gamma}\right)_{\gamma \in \ddot{\Gamma}_{\infty}},\left(\mathbf{X}_{t}\right)_{t \in \mathbb{R}_{+}}\right) .
$$


It follows directly from our construction that (8.28) is a realization of the independent infinite particle process,

For a fixed $\gamma \in \ddot{\Gamma}_{\infty}$, the finite-dimensional distributions of the process $\mathbf{X}_{t}$ under $\mathbf{P}_{\gamma}$ are given by

$$
\begin{aligned}
& \mathbf{P}_{\gamma}\left(\mathbf{X}_{t_{1}} \in A_{1}, \mathbf{X}_{t_{2}} \in A_{2}, \ldots, \mathbf{X}_{t_{n}} \in A_{n}\right) \\
&= \mathbf{P}_{\mathbf{x}}\left(\mathbf{X}_{t_{1}} \in A_{1}, \mathbf{X}_{t_{2}} \in A_{2}, \ldots, \mathbf{X}_{t_{n}} \in A_{n}\right) \\
&= \mathbb{P}_{\mathbf{x}}\left(\omega\left(t_{1}\right) \in I^{-1} A_{1}, \omega\left(t_{2}\right) \in I^{-1} A_{2}, \ldots, \omega\left(t_{n}\right) \in I^{-1} A_{n}\right) \\
&= \int_{I^{-1} A_{1}} \mathbb{P}_{t_{1}}\left(\mathbf{x}, d \mathbf{x}_{1}\right) \int_{I^{-1} A_{2}} \mathbb{P}_{t_{2}-t_{1}}\left(\mathbf{x}_{1}, d \mathbf{x}_{2}\right) \ldots \\
& \int_{I^{-1} A_{n}} \mathbb{P}_{t_{n}-t_{n-1}}\left(\mathbf{x}_{n-1}, d \mathbf{x}_{n}\right) \\
&= \int_{A_{1}} \mathbf{P}_{t_{1}}\left(\gamma, d \gamma_{1}\right) \int_{A_{2}} \mathbf{P}_{t_{2}-t_{1}}\left(\gamma_{1}, d \gamma_{2}\right) \cdots \int_{A_{n}} \mathbf{P}_{t_{n}-t_{n-1}}\left(\gamma_{n-1}, d \gamma_{n}\right), \\
& 0<t_{1}<t_{2}<\cdots<t_{n}, \quad A_{1}, A_{2}, \ldots, A_{n} \in \mathcal{B}\left(\ddot{\Gamma}_{\infty}\right)
\end{aligned}
$$

where $\mathbb{P}_{t}\left(\mathbf{x}_{i}, d \mathbf{x}_{j}\right):=\mathbb{P}_{t, \mathbf{x}_{i}}\left(d \mathbf{x}_{j}\right)$ and $\mathbf{x}$ is an arbitrary element of $I^{-1}\{\gamma\}$. Thus, the finite-dimensional distributions of $\mathbf{X}_{t}$ are determined by the Markov semigroup of kernels $\left(\mathbf{P}_{t}\right)_{t \in \mathbb{R}_{+}}$. Hence, it follows that (8.28) is a time homogeneous Markov process on $\left(\ddot{\Gamma}_{\infty}, \mathcal{B}\left(\ddot{\Gamma}_{\infty}\right)\right)$ with transition probability function $\left(\mathbf{P}_{t}\right)_{t \in \mathbb{R}_{+}}$ (see e.g. [8, Chapter 1, Section 3]).

Finally, we note that any measure on the space $(\boldsymbol{\Omega}, \mathbf{F})$ is uniquely determined by its finite-dimensional distributions, and therefore the constructed continuous Markov process is unique.

Remark 8.2. It is easy to see that the process $\left(\mathbf{X}_{t}\right)_{t \in \mathbb{R}_{+}}$constructed in the course of the proof of Theorem 8.1 is even Markov with respect to the filtration $\left(\mathbf{F}_{t+}\right)_{t \in \mathbb{R}_{+}}$, where $\mathbf{F}_{t+}:=\bigcap_{s>t} \mathbf{F}_{t}$.

The following corollary states that, if the dimension $d$ of the manifold $X$ is $\geq 2$, then the process $\mathbf{X}_{t}$ starting at $\gamma \in \Gamma_{\infty}$ lives with $\mathbf{P}_{\gamma}$-probability one in $\Gamma_{\infty}$, i.e., the particles never collide (compare with [35]).

Corollary 8.1. Let $\left(\mathrm{C}^{\prime}\right),(\mathrm{C} 2)$ and $(\mathrm{C} 3)$ hold, and let $d \geq 2$. Then, the independent infinite particle process can be realized as the unique continuous, time homogeneous Markov process

$$
\mathbf{M}=\left(\boldsymbol{\Omega}, \mathbf{F},\left(\mathbf{F}_{t}\right)_{t \in \mathbb{R}_{+}},\left(\boldsymbol{\theta}_{t}\right)_{t \in \mathbb{R}_{+}},\left(\mathbf{P}_{\gamma}\right)_{\gamma \in \Gamma_{\infty}},\left(\mathbf{X}_{t}\right)_{t \in \mathbb{R}_{+}}\right)
$$

on the state-space $\left(\Gamma_{\infty}, \mathcal{B}\left(\Gamma_{\infty}\right)\right)$ with transition probability function $\left(\mathbf{P}_{t}\right)_{t \in \mathbb{R}_{+}} \cdot$ 
Proof. First, we claim that, if $d \geq 2$, then

$$
P_{x_{1}} \otimes P_{x_{2}}\left(\exists t>0: \omega_{1}(t)=\omega_{2}(t)\right)=0, \quad x_{1}, x_{2} \in X
$$

i.e., two independent Brownian motions on $X$ never collide.

In the Euclidean case $X=\mathbb{R}^{d}$, this is a direct consequence of a classical result from potential theory. Indeed, $\omega_{1}(t / 2)-\omega_{2}(t / 2)$ is standard Brownian motion on $\mathbb{R}^{d}$ starting at $x_{1}-x_{2}$, and therefore (8.29) is equivalent to the equality

$$
P_{x_{1}-x_{2}}(\exists t>0: \omega(t)=0)=0,
$$

which is true since points are polar for Brownian motion on $\mathbb{R}^{d}$ if $d \geq 2$ (see e.g. [31, Proposition 2.5]).

In the general case, to prove (8.29) one can follow the idea of [35]. First, we note that $(8.29)$ is equivalent to

$$
P_{x_{1}} \otimes P_{x_{2}}\left(\exists t>\frac{1}{n}: \omega_{1}(t)=\omega_{2}(t)\right)=0 \quad \forall n \in \mathbb{N} .
$$

Using the Markov property, we have

$$
\begin{aligned}
P_{x_{1}} \otimes & P_{x_{2}}\left(\exists t>\frac{1}{n}: \omega_{1}(t)=\omega_{2}(t)\right) \\
= & \int_{X^{2}} m\left(d y_{1}\right) m\left(d y_{2}\right) p\left(\frac{1}{n}, x_{1}, y_{1}\right) p\left(\frac{1}{n}, x_{2}, y_{2}\right) \\
& \times P_{y_{1}} \otimes P_{y_{2}}\left(\exists t>0: \omega_{1}(t)=\omega_{2}(t)\right) .
\end{aligned}
$$

By virtue of (8.30) and (8.31), it suffices to verify that the equality (8.29) holds only for $m^{\otimes 2}$-a.a. $\left(x_{1}, x_{2}\right) \in X^{2}$.

There exists a countable, locally finite covering $\left\{U^{(i)}\right\}_{i=1}^{\infty}$ of the manifold $X$ such that each $U^{(i)}$ is an open set in $X$ diffeomorphic to the open cube $(-1,1)^{d}$ in $\mathbb{R}^{d}$. Furthermore, two independent Brownian motions on $X$ which start respectively at $x_{1}$ and $x_{2}$ form a Brownian motion on the manifold $X^{2}$ starting at the point $\left(x_{1}, x_{2}\right)$. Hence, our problem can be reduced to the following one: Show that

$$
\begin{gathered}
P_{\left(x_{1}, x_{2}\right)}\left(\exists t>0: \omega_{1}(t)=\omega_{2}(t) \in U_{j}^{(i)}\right)=0, \\
\quad \text { for } m^{\otimes 2} \text {-a.a. }\left(x_{1}, x_{2}\right) \in X^{2}, i, j \in \mathbb{N},
\end{gathered}
$$

where $U_{j}^{(i)}$ is the subset of $U^{(i)}$ that is diffeomorphic to the open cube

$$
\mathcal{C}_{j}:=\left(-1+(1+j)^{-1}, 1-(1+j)^{-1}\right)^{d} .
$$


Let us consider the Dirichlet form that corresponds to Brownian motion on $X^{2}$ :

$$
\begin{aligned}
\mathcal{E}(f, g):= & \int_{X^{2}}\left[\left\langle\nabla_{x_{1}}^{X} f\left(x_{1}, x_{2}\right), \nabla_{x_{1}}^{X} g\left(x_{1}, x_{2}\right)\right\rangle_{T_{x_{1}}(X)}\right. \\
& \left.+\left\langle\nabla_{x_{2}}^{X} f\left(x_{1}, x_{2}\right), \nabla_{x_{2}}^{X} g\left(x_{1}, x_{2}\right)\right\rangle_{T_{x_{2}}(X)}\right] m\left(d x_{1}\right) m\left(d x_{2}\right) .
\end{aligned}
$$

The bilinear form $\mathcal{E}$ is defined first for $f, g \in \mathcal{D}^{\otimes 2}=C_{0}^{\infty}\left(X^{2}\right)$, and then it is closed.

Since $m^{\otimes 2}\left(\left\{\left(x_{1}, x_{2}\right) \in X^{2}: x_{1}=x_{2}\right\}\right)=0$, to prove (8.32) it is enough to construct a sequence $\left\{u_{n}\right\}_{n=1}^{\infty} \subset \operatorname{Dom}(\mathcal{E})$ such that $u_{n}$ 's converge pointwisely to the indicator function of the set $\left\{\left(x_{1}, x_{2}\right) \in X^{2}: x_{1}=x_{2} \in U_{j}^{(i)}\right\}$ and $\sup _{n} \mathcal{E}\left(u_{n}, u_{n}\right)<\infty($ see $[35])$.

By using the representation of the Dirichlet form $\mathcal{E}$ in local coordinates on $U^{(i)}$, we get, for any function $f \in \operatorname{Dom}(\mathcal{E})$ having support in $\left(U^{(i)}\right)^{2}$ :

$$
\begin{aligned}
& \mathcal{E}(f, f)=\frac{1}{2} \int_{(-1,1)^{2 d}} \sum_{k, l=1}^{d}\left[g^{k l}\left(x_{1}\right) \frac{\partial f}{\partial x_{1}^{k}}\left(x_{1}, x_{2}\right) \frac{\partial f}{\partial x_{1}^{l}}\left(x_{1}, x_{2}\right)\right. \\
& \left.+g^{k l}\left(x_{2}\right) \frac{\partial f}{\partial x_{2}^{k}}\left(x_{1}, x_{2}\right) \frac{\partial f}{\partial x_{2}^{l}}\left(x_{1}, x_{2}\right)\right] \sqrt{\mathbf{g}\left(x_{1}\right)} \sqrt{\mathbf{g}\left(x_{2}\right)} d x_{1}^{1} \cdots d x_{1}^{d} d x_{2}^{1} \cdots d x_{2}^{d},
\end{aligned}
$$

where $\mathbf{g}$ denotes the determinant of the matrix $\left(g_{k l}\right)_{k, l=1}^{d}:=\left(\left\langle\partial / \partial x^{k}\right.\right.$, $\left.\left.\partial / \partial x^{l}\right\rangle\right)_{k, l=1}^{d}$, and $\left(g^{k l}\right)_{k, l=1}^{d}$ is its inverse. We conclude from (8.34) that there exists a constant $C>0$ such that, for each function $f \in \operatorname{Dom}(\mathcal{E})$ having support in a fixed $\left(U_{j}^{(i)}\right)^{2}$,

$$
\mathcal{E}(f, f) \leq C \mathcal{E}_{\mathrm{E}}(f, f),
$$

where $\mathcal{E}_{\mathrm{E}}$ is the (Euclidean) Dirichlet form on $\mathbb{R}^{2 d}$ :

$$
\mathcal{E}_{\mathrm{E}}(f, f)=\int_{\mathbb{R}^{2 d}}\left\langle\nabla f\left(x_{1}, x_{2}\right), \nabla f\left(x_{1}, x_{2}\right)\right\rangle d x_{1}^{1} \cdots d x_{1}^{d} d x_{2}^{1} \cdots d x_{2}^{d},
$$

$\nabla$ denoting the usual gradient on $\mathbb{R}^{2 d}$. Hence, it suffices to construct for any fixed $j \in \mathbb{N}$ a sequence $\left\{u_{n}\right\}_{n=1}^{\infty} \subset \operatorname{Dom}\left(\mathcal{E}_{\mathrm{E}}\right)$ such that each $u_{n}$ has support in $\mathcal{C}_{j+1}^{2}$, the $u_{n}$ 's converge pointwisely to the indicator of the set $\left\{\left(x_{1}, x_{2}\right) \in \mathbb{R}^{2 d}\right.$ : $\left.x_{1}=x_{2} \in \mathcal{C}_{j}\right\}$, and $\sup _{n} \mathcal{E}_{\mathrm{E}}\left(u_{n}, u_{n}\right)<\infty$. But the existence of such sequence can be seen by a trivial modification of the proof of Proposition 1 in [35]. Thus, (8.32) and hence also the claim (8.29) are proven.

The rest of the proof follows from that of Theorem 8.1. Instead of the set A given by (8.25), one should use its subset

$$
\mathbf{A}^{\prime}:=\mathbf{A} \cap\left[\bigcap_{\{i, j\} \subset \mathbb{N}}\left\{\omega_{i}(t) \neq \omega_{j}(t) \forall t \in \mathbb{R}_{+}\right\}\right]
$$


By (8.7), (8.26), (8.29) and (8.35), we get

$$
\mathbb{P}_{\mathbf{x}}\left(\mathbf{A}^{\prime}\right)=1
$$

for each $\mathbf{x} \in \widetilde{X}^{\mathbb{N}}$ satisfying (8.6), so that the measure $\mathbb{P}_{\mathbf{x}}$ can be considered as a probability measure on $\left(\mathbf{A}^{\prime}, \mathcal{B}\left(\mathbf{A}^{\prime}\right)\right)$. Finally, noting that

$$
\mathbf{I A}^{\prime} \subset C\left(\mathbb{R}_{+} ; \Gamma_{\infty}\right)
$$

(compare with Lemma 8.7), we get the corollary by a corresponding modification of the last part of the proof of Theorem 8.1.

Remark 8.3 (Path-wise construction of the independent particle process on $\left.\Gamma_{\infty}\right)$. Let $d \geq 2$ and let us consider the probability space $\left(\boldsymbol{\Omega}, \mathcal{C}_{\sigma}(\boldsymbol{\Omega}), \mathbf{P}\right)$, where $\boldsymbol{\Omega}:=\Omega^{X}, \Omega:=C\left(\mathbb{R}_{+} ; X\right), \mathcal{C}_{\sigma}(\boldsymbol{\Omega})$ is the product $\sigma$-algebra on $\boldsymbol{\Omega}$ constructed from the $\sigma$-algebra $\mathcal{F}$ on $\Omega$ defined by (8.1), and $\mathbf{P}:=\bigotimes_{x \in X} P_{x}$. For any fixed $\gamma \in \Gamma_{\infty}$ and $t \in \mathbb{R}_{+}$, we define

$$
\boldsymbol{\Omega} \ni \omega=\left(\omega_{x}\right)_{x \in X} \mapsto \mathbf{X}_{t}^{\gamma}(\omega):=\sum_{x \in \gamma} \varepsilon_{X_{t}^{x}(\omega)},
$$

where $X_{t}^{x}(\omega):=\omega_{x}(t)$. Thus, for any $\gamma \in \Gamma_{\infty}$ we have constructed a process $\mathbf{X}^{\gamma}:=\left(\mathbf{X}_{t}^{\gamma}\right)_{t \in \mathbb{R}_{+}}$which takes values in the space of all measures on $X$. Let us fix any $\mathbf{x}=\left(x_{k}\right)_{k=1}^{\infty} \in I^{-1}\{\gamma\}$. Then, $\mathbb{P}_{\mathbf{x}}=\mathbf{P} \circ \mathbf{I}_{\mathbf{x}}^{-1}$, where $\mathbb{P}_{\mathbf{x}}$ is defined by (8.7) and

$$
\boldsymbol{\Omega} \ni \omega=\left(\omega_{x}\right)_{x \in X} \mapsto \mathbf{I}_{\mathbf{x}} \omega:=\left(\omega_{x_{k}}\right)_{k=1}^{\infty} \in \Omega^{\mathbb{N}} .
$$

Hence, it follows from the proof of Theorem 8.1 (respectively Corollary 8.1) that with $\mathbf{P}$ probability one the independent particle process $\mathbf{X}^{\gamma}$ starts at $\gamma$, never leaves $\Gamma_{\infty}$, i.e., $\mathbf{P}\left(\forall t>0: \mathbf{X}_{t}^{\gamma} \in \Gamma_{\infty}\right)=1$, and has sample paths which are continuous in the $d_{\infty}$ metric.

In the case $d=1$, in order to give a corresponding path-wise construction of the independent particle process on $\ddot{\Gamma}_{\infty}$, we proceed as follows. We consider the probability space $\left(\boldsymbol{\Omega}, \mathcal{C}_{\sigma}(\boldsymbol{\Omega}), \mathbf{P}\right)$, where

$$
\boldsymbol{\Omega}:=\underset{n(\cdot) \in \mathbb{N}^{X}}{\times} \underset{x \in X}{\times} \Omega^{n(x)}
$$

$\mathcal{C}_{\sigma}(\boldsymbol{\Omega})$ is the corresponding product $\sigma$-algebra on $\boldsymbol{\Omega}$, and

$$
\mathbf{P}:=\bigotimes_{n(\cdot) \in \mathbb{N}^{X}} \bigotimes_{x \in X} P_{x}^{\otimes n(x)}
$$

For any $\gamma \in \ddot{\Gamma}_{X}$, we define $\hat{\gamma} \in \Gamma_{X}$ and a mapping $n_{\gamma}: \hat{\gamma} \rightarrow \mathbb{N}$ by

$$
\hat{\gamma}:=\operatorname{supp} \gamma, \quad \hat{\gamma} \ni x \mapsto n_{\gamma}(x):=\gamma(\{x\}) \in \mathbb{N} .
$$


We extend the mapping $n_{\gamma}(\cdot)$ to the whole of $X$ by $n_{\gamma}(x):=1$ for all $x \in X \backslash \hat{\gamma}$. Now, for any fixed $\gamma \in \ddot{\Gamma}_{\infty}$, we define a process $\mathbf{X}^{\gamma}:=\left(\mathbf{X}_{t}^{\gamma}\right)_{t \in \mathbb{R}_{+}}$setting for each $t \in \mathbb{R}_{+}$

$$
\boldsymbol{\Omega} \ni \omega=\left(\omega_{n(\cdot), x}^{1}, \ldots, \omega_{n(\cdot), x}^{n(x)}\right)_{n(\cdot) \in \mathbb{N}^{X}, x \in X} \mapsto \mathbf{X}_{t}^{\gamma}(\omega):=\sum_{x \in \hat{\gamma}} \sum_{i=1}^{n_{\gamma}(x)} \varepsilon_{\omega_{n_{\gamma}(\cdot), x}^{i}(t)} .
$$

Analogously to the above, we conclude that with $\mathbf{P}$ probability one the independent particle process $\mathbf{X}^{\gamma}$ starts at $\gamma$, never leaves $\ddot{\Gamma}_{\infty}$, and has sample paths continuous in the $d_{\infty}$ metric.

Remark 8.4. The Markov process on the state space $\Gamma_{\infty}$ that was constructed in Corollary 8.1 is a strong Markov process. This can be shown by a modification of the proof of [12, Theorem 5.10] using Corollary 6.2. Furthermore, by proving a corresponding Feller property of $\mathbf{P}_{t}$ on $\ddot{\Gamma}_{\infty}$ with respect to the metric $d_{1}$, one can show that the Markov process on the state space $\ddot{\Gamma}_{\infty}$ that was constructed in Theorem 8.1 also possesses the strong Markov property.

\section{$\S 9 . \quad$ Appendix: Proof of Lemma 8.1}

Let us fix $r>0, \delta>0, x \in X, 0 \leq t_{1}<\cdots<t_{n}, n \geq 2$, with $t_{n}-t_{1} \leq \delta$. Let

$$
A:=\left\{\omega \in \Omega: d\left(\omega\left(t_{1}\right), \omega\left(t_{j}\right)\right)>r \text { for some } j=2, \ldots, n\right\},
$$

and let us show that

$$
P_{x}(A) \leq 2 \tau\left(\delta, \frac{1}{2} r\right)
$$

Let

$$
\begin{aligned}
& B:=\left\{\omega \in \Omega: d\left(\omega\left(t_{1}\right), \omega\left(t_{n}\right)\right)>\frac{1}{2} r\right\}, \\
& C_{j}:=\left\{\omega \in \Omega: d\left(\omega\left(t_{j}\right), \omega\left(t_{n}\right)\right)>\frac{1}{2} r\right\}, \\
& D_{j}:=\left\{\omega \in \Omega: d\left(\omega\left(t_{1}\right), \omega\left(t_{j}\right)\right)>r\right. \\
&\left.\quad \text { and } d\left(\omega\left(t_{1}\right), \omega\left(t_{k}\right)\right) \leq r \text { for } k=1, \ldots, j-1\right\} .
\end{aligned}
$$

Then,

$$
A \subset B \cup\left[\bigcup_{j=1}^{n}\left(C_{j} \cap D_{j}\right)\right]
$$


Therefore,

$$
P_{x}(A) \leq P_{x}(B)+\sum_{j=1}^{n} P_{x}\left(C_{j} \cap D_{j}\right) .
$$

Define $\widetilde{D}_{j} \subset X^{j}, \widetilde{C}_{j} \subset X^{2}$ by

$$
\begin{aligned}
\widetilde{D}_{j}:= & \left\{\left(x_{1}, \ldots, x_{j}\right) \in X^{j}: d\left(x_{1}, x_{j}\right)>r\right. \\
& \text { and } \left.d\left(x_{1}, x_{k}\right) \leq r \text { for } k=1, \ldots, j-1\right\}, \\
\widetilde{C}_{j}:= & \left\{\left(x_{1}, x_{2}\right) \in X^{2}: d\left(x_{1}, x_{2}\right)>\frac{1}{2} r\right\} .
\end{aligned}
$$

Then,

$$
\begin{aligned}
P_{x}\left(C_{j} \cap D_{j}\right)= & \int_{X} \cdots \int_{X} p\left(t_{1}, x, d x_{1}\right) \cdots p\left(t_{j}-t_{j-1}, x_{j-1}, d x_{j}\right) \\
& \times p\left(t_{n}-t_{j}, x_{j}, d x_{n}\right) 1_{\widetilde{D}_{j}}\left(x_{1}, \ldots, x_{j}\right) 1_{\widetilde{C}_{j}}\left(x_{j}, x_{n}\right) \\
\leq & \tau\left(\delta, \frac{1}{2} r\right) \int_{X} \cdots \int_{X} p\left(t_{1}, x, d x_{1}\right) \cdots \\
& p\left(t_{j}-t_{j-1}, x_{j-1}, d x_{j}\right) 1_{\widetilde{D}_{j}}\left(x_{1}, \ldots, x_{j}\right) \\
= & \tau\left(\delta, \frac{1}{2} r\right) P_{x}\left(D_{j}\right) .
\end{aligned}
$$

Since the sets $D_{j}$ are disjoint, we have

$$
\sum_{j=1}^{n} P_{x}\left(C_{j} \cap D_{j}\right) \leq \sum_{j=1}^{n} \tau\left(\delta, \frac{1}{2} r\right) P_{x}\left(D_{j}\right) \leq \tau\left(\delta, \frac{1}{2} r\right),
$$

and since $P_{x}(B) \leq \tau(\delta,(1 / 2) r)$, we get $(9.2)$.

It follows from $(9.1),(9.2)$ that

$$
P_{x}\left(d\left(\omega\left(t_{j}\right), \omega\left(t_{k}\right)\right)>2 r \text { for some } j, k, 1 \leq j, k \leq n\right) \leq 2 \tau\left(\delta, \frac{1}{2} r\right) .
$$

Indeed, if $d\left(\omega\left(t_{j}\right), \omega\left(t_{k}\right)\right)>2 r$, then $d\left(\omega\left(t_{1}\right), \omega\left(t_{j}\right)\right)>r$ or $d\left(\omega\left(t_{1}\right), \omega\left(t_{k}\right)\right)>r$.

Since the estimate (9.3) is independent of $n$ and $t_{1}, \ldots, t_{n}$, we get

$$
P_{x}\left(d\left(\omega\left(t_{1}\right), \omega\left(t_{2}\right)\right)>2 r \text { for some } t_{1}, t_{2} \in \mathbb{R}_{+} \cap \mathbb{Q},\left|t_{1}-t_{2}\right| \leq \delta\right) \leq 2 \tau\left(\delta, \frac{1}{2} r\right) .
$$

Due to the continuity of the trajectories $\omega \in \Omega,(9.4)$ implies the statement of the lemma. 


\section{Acknowledgements}

We thank Tobias Kuna for useful discussions. The authors were partially supported by the SFB 256, Bonn University, and SFB 343, Bielefeld University. The support of the DFG through Projects 436 113/39 and 436 113/43, and of the BMBF through Project UKR-004-99 is gratefully acknowledged.

\section{References}

[1] Albeverio, S., Daletskii, A. and Lytvynov, E., Laplace operators and diffusions in tangent bundles over Poisson spaces, Infinite Dimensional Stochastic Analysis (Ph. Clément et al., Eds.), pp. 1-24, Korink. Nederl. Akad. Wetensch., Amsterdam, 2000.

[2] — Laplace operators on differential forms over configuration spaces, J. Geom. Phys., 37 (2001), 15-46.

[3] Albeverio, S., Kondratiev, Yu. G. and Röckner, M., Differential geometry of Poisson spaces, C. R. Acad. Sci. Paris, 323 (1996), 1129-1134.

[4] - Canonical Dirichlet operator and distorted Brownian motion on Poisson spaces, C. R. Acad. Sci. Paris, 323 (1996), 1179-1184.

[5] - Analysis and geometry on configuration spaces, J. Funct. Anal., 154 (1998), 444-500.

[6] - Analysis and geometry on configuration spaces: The Gibbsian case, J. Funct. Anal., 157 (1998), 242-291.

[7] Berezansky, Yu. M., Kondratiev, Yu. G., Kuna, T. and Lytvynov, E., On a spectral representation for correlation measures in configuration space analysis, Meth. Funct. Anal. Topol., 5 (1999), no. 4, 87-100.

[8] Blumenthal, R. M., Excursions of Markov Processes, Birkhäuser, Boston, Basel, Berlin, 1992.

[9] Blumenthal, R. M. and Getoor, R. K., Markov Processes and Potential Theory, Academic Press, New York, London, 1968.

[10] Davies, E. B., Heat Kernels and Spectral Theory, Cambridge Univ. Press, Cambridge, 1989.

[11] Doob, J. L., Stochastic Processes, Jhon Wiley \& Sons, New York, London, 1953.

[12] Dynkin, E. B., Theory of Markov Processes, Pergamon Press, Oxford, London, New York, Paris, 1960.

[13] Fukushima, M., Dirichlet Forms and Symmetric Markov Processes, North-Holland, Amsterdam, 1980.

[14] Grigor'yan, A., Heat kernel upper bounds on a complete non-compact manifold, Rev. Mat. Iberoamericana, 10 (1994), 395-452.

[15] — - Heat kernel of a noncompact Riemannian manifold, Proc. Sympos. Pure Math., 57, pp. 239-262, American Math. Soc., 1995.

[16] Hirai, T. and Shimomura, H., Relations between unitary representations of diffeomorphism groups and those of the infinite symmetric group of related permutation group, J. Math. Kyoto Univ., 37 (1997), 261-316.

[17] Kallenberg, O., Random Measures, Akedemie-Verlag, Berlin, 4th edition, 1986.

[18] Kondratiev, Yu. G. and Kuna, T., Harmonic analysis on configuration space I. General theory, Infin. Dimens. Anal. Quantum Prob. Relat. Top., 5 (2002), 201-233.

[19] - Harmonic analysis on configuration space II. Gibbs states, in preparation, 2000.

[20] — Non-equilibrium dynamics of infinite particle systems and the Bogolubov hierarchy, in preparation, 2000.

[21] Kondratiev, Yu. G. and Skorohod, A. V., Stochastic analysis on configuration spaces, to appear in ROSE. 
[22] Kuna, T., Studies in Configuration Space Analysis and Applications, Ph.D. Thesis, Bonn University, 1999.

[23] Lenard, A., Correlation functions and the uniqueness of the state in classical statistical mechanics, Comm. Math. Phys., 30 (1972), 35-44.

[24] - States of classical statistical mechanical systems of infinitely many particles. I., Arch. Ration. Mech. Anal., 59 (1975), 219-239.

[25] - States of classical statistical mechanical systems of infinitely many particles. II. Characterization of correlation measures, Arch. Ration. Mech. Anal., 59 (1975), 241256.

[26] Macchi, O., The coincidence approach to stochastic point processes, Adv. Appl. Prob., 7 (1975), 83-122.

[27] Ma, Z.-M. and Röckner, M., Introduction to the Theory of (Non-Symmetric) Dirichlet Forms, Springer-Verlag, Berlin, New York, 1992.

[28] — Construction of diffusions on configuration spaces, SFB 343 Preprint, Univ. Bielefeld, 1998, Osaka J. Math., 37 (2000), 273-314.

[29] Matthes, K., Kerstan, J. and Mecke, J., Infinite Divisible Point Processes, AkademieVerlag, Berlin, 1978.

[30] Nelson, E., Feynman integrals and the Schrödinger equation, J. Math. Phys., 5 (1964), 332-343.

[31] Port, S. C. and Stone, C. J., Brownian Motion and Classical Potential Theory, Academic Press, New York, London, 1978.

[32] Rachev, S. T., Probability Metrics and the Stability of Stochastic Models, Jhon Wiley \& Sons, Chichester, New York, 1991.

[33] Röckner, M., Stochastic analysis on configuration spaces: Basic ideas and recent results, In: New Directions in Dirichlet Forms. J. Jost et al. (eds.), Stud. Adv. Math., 8, American Math. Soc., 1998, 157-232.

[34] Röckner, M. and Schied, A., Rademacher's theorem on configuration spaces and applications, J. Funct. Anal., 169 (1999), 325-356.

[35] Röckner, M. and Schmuland, B., A support property for infinite-dimensional interacting diffusion processes, C. R. Acad. Sci. Paris, Série I, 326 (1998), 359-364.

[36] Shiga, T. and Takanashi, Y., Ergodic properties of the equilibrium process associated with infinitely many Markovian particles, Publ. RIMS, Kyoto Univ., 9 (1974), 505-516.

[37] Shimomura, H., Poisson measures on the configuration space and unitary representations of the group of diffeomorphisms, J. Math. Kyoto Univ., 34 (1994), 599-614.

[38] Takeda, M., On a martingale method for symmetric diffusion process and its applications, Osaka J. Math., 26 (1989), 605-623.

[39] Vershik, A. M., Gelfand, I. M. and Graev, M. I., Representations of the group of diffeomorphisms, Russian Math. Surveys, 30 (1975), no. 6, 1-50. 